\title{
ON THE POSSIBILITY OF INFORMATIONALLY EFFICIENT MARKETS
}

\author{
Xavier Vives
}


The Public-Private Sector Research Center is a Research Center based at IESE Business School. Its mission is to develop research that analyses the relationships between the private and public sectors primarily in the following areas: regulation and competition, innovation, regional economy and industrial politics and health economics.

Research results are disseminated through publications, conferences and colloquia. These activities are aimed to foster cooperation between the private sector and public administrations, as well as the exchange of ideas and initiatives.

The sponsors of the Public-Private Sector Research Center are the following:

- Ajuntament de Barcelona

- Departament d' Economia i Coneixement de la Generalitat de Catalunya

- Departament d' Empresa i Ocupació de la Generalitat de Catalunya

- Diputació de Barcelona

- Fundació AGBAR

- Institut Català de les Empreses Culturals (ICEC)

- PricewaterhouseCoopers

- Sanofi

- FGC

The contents of this publication reflect the conclusions and findings of the individual authors, and not the opinions of the Center's sponsors. 


\title{
On the Possibility of Informationally Efficient Markets
}

\author{
Xavier Vives* \\ IESE Business School
}

June 2014

\begin{abstract}
This paper presents conditions for a resolution of the Grossman-Stiglitz paradox of informationally efficient markets. We display a market with asymmetric information where a privately revealing equilibrium obtains in a competitive framework and where incentives to acquire information are preserved as long as the correlation in traders' valuations is not too large. The equilibrium is efficient, and the problems associated with fully revealing rational expectations equilibria are precluded without resorting necessarily to noise traders. The robustness of the results to general information structures is established and the effect of market power is examined in a large market approximation to the competitive economy. The model is applied to explain changes in bidding behavior in central bank liquidity auctions in the crisis period.
\end{abstract}

Keywords: adverse selection, information acquisition, double auction, multi-unit auctions, rate of convergence, market power, complementarities, liquidity and Treasury auctions

JEL Codes: D82, D84, G14, E59

This paper was delivered as the Schumpeter lecture in the $28^{\text {th }}$ Congress of the EEA in Gothenburg in 2013. The comments of the Editor Dirk Bergemann and three anonymous referees have contributed to improve the paper. I am grateful to Carolina Manzano, Bruno Biais, Paul Klemperer, Jaume Ventura and to participants at presentations of early versions of the paper (with title "A Large-Market Rational Expectations Equilibrium Model”) at ESEM in Oslo (August 2011), ESSET (July 2012), Arizona State University and Caltech (April 2012) for helpful comments. I thank also Anna Bayona, Rodrigo Escudero and Jorge Paz for excellent research assistance. The research reported in this paper has been supported by European Advanced Grant (no. 230254). Complementary support from project ECO2011-29533 of the Spanish Ministry of Education and Science at the Public-Private Sector Research Center at IESE is acknowledged. 


\section{Introduction}

The financial crisis has questioned the informational efficiency of the market, popularized as the "efficient market hypothesis". ${ }^{1}$ Indeed, commentators point at bubbles in stock markets (e.g. tech bubble) and in real estate markets as evidence of the demise of the hypothesis. A basic problem in an informationally efficient market was pointed out by Grossman and Stiglitz (1976, 1980). As stated plainly by John Kay (FT July 16, 2013): "A contradiction lies at the heart of the efficient market hypothesis: if market prices did incorporate all available information about the value of an asset, no one would have an incentive to obtain that information in the first place". The implications of the lack of market informational efficiency are important for investment decisions, accounting (mark to market), and managerial incentives. We keep relying on prices, however. When prices for CDS contracts shoot up for banks, firms, or countries we infer trouble ahead (in terms of an increase in probability of default); when interbank spreads spike we infer liquidity and solvency problems for banks. A question therefore is whether the reliance on prices is warranted and how information issues help explain behavior by market participants during a crisis.

Rational expectations models have proved to be a workhorse for the analysis of situations involving uncertainty and private information. An important aim has been to provide a workable model of Hayek's (1945) idea that prices aggregate the dispersed information of agents in the economy, given prices' dual role as index of scarcity and conveyors of information. However, the concept of rational expectations equilibrium (REE) is not without problems - and this is especially true of fully revealing REE in competitive markets. ${ }^{2}$ The concept has two main difficulties. First, the above mentioned Grossman-Stiglitz paradox: If information is costly and prices are fully revealing, and if the traders perceive that they cannot affect the informational content of prices, then at equilibrium no trader pays to gather information because all information (including his) is

\footnotetext{
1 "For more than four decades, financial markets and the regulations that govern them were underpinned by what is known as the efficient markets hypothesis. All that changed after the financial crisis". (Norma Cohen, FT, Jan 24, 2012)

2 The concept of a fully revealing REE is relevant since, absent other frictions, this equilibrium is Pareto optimal.
} 
already freely available in the prices. However, if no trader gathers information, then the prices cannot convey information; and if prices convey no information and information is sufficiently cheap then some trader will want to acquire information. In those circumstances there is no equilibrium and fully revealing equilibrium prices are logically impossible (Grossman and Stiglitz (1976, 1980); Matthews (1984), Jackson (2003) for auctions). Second, the equilibrium need not be implementable; that is, it may not be possible to find a trading mechanism (in a well-specified game) that delivers the fully revealing REE. An added problem arises if the competitive REE is defined in a finiteagent economy, since then traders realize that prices convey information but do not realize the impact of their actions on the price (this is the "schizophrenia" problem of Hellwig (1980)). These problems are typically overcome by considering noisy REE in large economies. Indeed, noise traders in competitive models have prevented trade from collapsing. ${ }^{3}$

This paper presents a simple, competitive, large-market model in which the valuation of each trader has a common and a private value component and where prices aggregate information efficiently. A key ingredient is that each trader receives a private signal which provides "bundled" information for both the common and the private value components. It shows how to obtain a privately revealing equilibrium in a well-specified game where each trader submits a demand schedule and has incentives to rely on his private signal and on the price. The information structure is taken from Vives (2011) which considers instead strategic supply competition and provides a finite-trader counterpart to the model in this paper. In a privately revealing equilibrium the price and the private signal of a trader are sufficient statistics for the pooled information of all traders in the market. The equilibrium is efficient, preserves incentives to acquire information provided that the common value component of the valuation is not overwhelming, and overcomes the problems of fully revealing REE without reliance on noise trading. Therefore, our equilibrium provides the conditions for a resolution of the Grossman-Stiglitz paradox. Furthermore, the Bayesian equilibrium in demand schedules obtained in the large market is not an artifact of the continuum specification for traders.

See, for example, Diamond and Verrecchia (1981) and Admati (1985). 
We verify that the large limit market equilibrium approximates well large finite markets equilibria, as studied in Vives (2011), in which traders are strategic and have incentives to influence prices, provided that the limit equilibrium calls for either positive or no information acquisition. This delivers a foundation for REE in the context of the model presented.

The valuations of traders have typically both common and idiosyncratic components. This applies, for example, to banks bidding for liquidity in central bank auctions or to Treasury auctions. In many occasions the signals traders receive provide bundled information for the common and the private value components. For example, when a bank bids for liquidity it will have in mind a resale value which is idiosyncratic to the bank, but related to the values of other banks, since the bank will transact typically in a secondary over-the-counter market. The value for the bank will reflect therefore this resale value and its liquidity needs, and the bank will have an imperfect assessment of its valuation. ${ }^{4}$

The model is of the linear-normal variety, as in Grossman and Stiglitz (1980), and it assumes declining marginal valuations. It is quite tractable and allows us to address the case of a good with an elastic exogenous supply as well as the case of a double auction; in addition, it enables us to characterize explicitly not only information acquisition but also rates of convergence of finite markets to the continuum limit. The model admits interpretation in terms of both financial markets and markets for goods.

We find that there is a unique linear equilibrium. In equilibrium, a high price indicates a high valuation, and this reduces responsiveness to price when there is private information. Indeed, demand schedules in this case are steeper and there is a greater

4 In a Treasury auction, bidders may have private information related to different expectations about the future resale value of the securities (e.g., different beliefs concerning how future inflation will affect nominally denominated securities) and to the idiosyncratic liquidity needs of traders. The common value component will tend to be more significant in Treasury auctions than in central bank auctions, since the main dealers buy Treasury bills primarily for resale. However, Hortaçsu and Kastl (2012) cannot reject the hypothesis that bidders in Canadian 3-month T-bill auctions have private values. 
extent of adverse selection in the market, which increases with the correlation of valuations and the noise in the signals. If the information effect is large enough, demand schedules may be upward sloping. Demand, as long as it is downward sloping, becomes steeper also as the slope of marginal valuation is steeper and as the slope of exogenous supply is flatter.

If the signals are costly to acquire and if traders face a convex cost of acquiring precision, then there is an upper bound on the correlation of valuation parameters below which there are incentives to purchase some precision. This upper bound is decreasing in the precision of the prior and in the marginal cost of acquiring precision; this bound is 1 - that is, perfect correlation - when the marginal cost (at zero precision) of acquiring precision is zero or when the prior is diffuse. A more diffuse prior or less correlation among valuations induces more effort to acquire information, and this effort is, in fact, socially optimal. The parameter region where the Grossman-Stiglitz paradox is maintained increases as correlation increases and we approach the common value case. 5 The result is robust to general normal information structures which can decentralize any REE outcome in the linear-quadratic model considered in this paper as studied by Bergemann et al. (2014). ${ }^{6}$ It is found that the Grossman-Stiglitz paradox parameter region grows with the strength of the common component in the signal.

The continuum economy is an idealization that allows solving Hellwig's trader schizophrenia problem since in the limit economy price-taking behavior is individually optimal. We check how large a market is needed for the equilibrium in the continuum economy to approximate well a finite market. The rate at which equilibria in finite replica markets (with $n$ traders and corresponding exogenous supply) approach the equilibrium in the continuum economy is $1 / \sqrt{n}$, the same rate at which the average signal of the traders tends to the limit average valuation parameter. We study the equilibrium of the

\footnotetext{
5 It is worth noting that if we do not insist on the market equilibrium being implementable, and consider the fully revealing equilibrium where traders have no private information, then equilibrium exists for all parameter values.

6 It applies also to noisy information structures where traders are exposed to an aggregate risk factor (as in Manzano and Vives (2011), for example).
} 
covert information acquisition game where traders choose information precisions and demand schedules simultaneously. ${ }^{7}$ The convergence results extend to the endogenous information acquisition case as long as the equilibrium in the continuum economy calls for a positive purchase or for no purchase of information. However, when this is not the case it may happen that the limit of equilibria with positive information acquisition in finite economies as the market grows large has no information purchase but this is not an equilibrium in the continuum economy. ${ }^{8}$ Market power may induce information acquisition in a finite large market when in the continuum market there would be no equilibrium. The underlying technical reason is that market power is not jointly continuous in the precision of information and the number of traders when evaluated at a zero precision.

The model developed here can be applied to explaining how banks bid for liquidity in central bank auctions (or how bidders behave in Treasury auctions). In particular, the model can be used to simulate the impact of a financial crisis on central bank liquidity auctions. We will see how adverse selection in a context where auction prices are informative may explain the fact that aggregate bid demands became much steeper after the subprime crisis episode in 2007 or Lehman Brothers failure in 2008.

There have been several attempts to resolve the Grossman-Stiglitz paradox in a common value environment. The most popular approach is to include noise traders who make prices not fully revealing as in Grossman and Stiglitz (1980), Hellwig (1980) or Admati (1985). ${ }^{9}$ This approach has been refined with the consideration of endowment shocks to traders (Diamond, and Verrecchia (1981), Verrecchia (1982), Ganguli and

7 This is irrelevant in the continuum market (except for the case of perfect correlation of values) but the consideration of a two-stage game where information acquisition is observable, may make a difference for the convergence results, particularly when in the continuum market there is no equilibrium with positive information acquisition.

8 Even in this case there is a fully revealing equilibrium, where traders have no private information in the continuum market. This equilibrium is not implementable. Therefore, interestingly, this equilibrium can be rationalized by demand function equilibria of large but finite economies in which traders purchase a vanishing amount of information.

9 According to Black (1986) noise trading "makes financial markets possible" and provides incentives "for people to seek out costly information which they will trade on". 
Yang (2009), Manzano and Vives (2011)), and uncertainty with larger dimension than prices (Allen (1981), Ausubel (1990)). In this approach it is found that there is strategic substitutability in information acquisition. ${ }^{10}$

Another line of attack has been to consider traders with market power in models with a finite number of traders. This is the case of Kyle (1989) and Jackson (1991) studying demand submission games. Kyle (1989) shows that equilibrium is well defined when noise trading is nonzero and as this noise vanishes prices do not become fully revealing because informed agents trade smaller and smaller amounts. Jackson (1991) shows the possibility of fully revealing prices with costly information acquisition, under some specific parametric assumptions, because traders realize that their actions influence prices. Kovalenkov and Vives (2014) show that risk neutral competitive traders would not enter a market and become informed (a variant of the Grossman-Stiglitz paradox). But if they are strategic, as the market grows large (parameterized by the amount of noise trading) the number of informed traders grows less than proportionately than the size of the market. Then prices become fully revealing because the aggregate response to private information grows even faster than noise trading and the incentives to acquire information are preserved.

Still, other work assumes non-expected utility traders: Krebs (2007) and Muendler (2007) resolve the paradox with schizophrenic agents who take into account the impact on market prices when choosing the quality of their information but not when they trade in the asset market; Condie and Ganguli (2011) and Mele and Sangiorgi (2009) consider ambiguity-averse traders.

In relation to the literature we present a competitive model, without resort to noise trading, with an equilibrium in which prices aggregate information efficiently, and where agents behave consistently both when acquiring information and trading. That is, we provide a resolution to the Grossman-Stiglitz paradox without having to assume noisy

10 The strategic substitutability result is robust in a model with endowment shocks which induce multiple equilibria (Manzano and Vives (2011)). 
prices. Our approach is based on an empirically plausible information structure with valuations with common and private value components and signals which provide bundled information about both components. This information structure is robust.

This paper is also related to the literature of information aggregation in auctions, Cournot markets, and markets in which traders compete in demand and/or supply functions. First, it is related to work on information aggregation, and on the foundations of REE in auction games, that developed from the pioneering studies of Wilson (1977) and Milgrom (1981) and have more recently been extended by Pesendorfer and Swinkels (1997). The convergence to price taking and to efficiency as double auction markets grow large has been analyzed in Wilson (1985), Satterthwaite and Williams (1989), and Rustichini, Satterthwaite, Williams (1994), and Cripps and Swinkels (2006). Our results on the model's double auction version are more closely related to Reny and Perry (2006), who present a double auction model with unit bids with a unique and privately revealing REE that is implementable as Bayesian equilibrium, and also offer an approximation in a finite large market. Given the nature of our own model, the results presented here deal with multi-unit demands and enable characterizations of an equilibrium's comparative static properties and of information acquisition. Furthermore, the model allows us to study convergence rates and to analyze the effect of an exogenous supply of the good. Second, a parallel literature on information aggregation has developed in the context of Cournot markets (Palfrey (1985); Vives (1988)). Dynamic extensions of the models have allowed fully revealing prices, but with a temporal lag, not allowing agents to condition their demands on current prices (e.g. learning from past prices; Hellwig (1982), Dubey, Geanakoplos and Shubik (1987), Vives (1988)). ${ }^{11}$

Third, the paper is closely related to the literature on strategic competition in terms of schedules in uniform price auctions developed from the seminal work of Wilson (1979) and Kyle (1989) (see also Wang and Zender (2002)). As stated, Vives (2011) considers strategic supply competition and provides a finite-trader counterpart to the

11 See also Golosov et al. (2014) for a dynamic decentralized foundation of fully revealing equilibria. 
model in this paper. ${ }^{12}$ It is obtained that the rate of convergence to price-taking behavior is as $1 / n$, which is faster than the rate of convergence of prices to the equilibrium in the continuum economy $1 / \sqrt{n}$ in the present paper.

The balance of the paper is organized as follows. Section 2 presents the model. Section 3 summarizes the problems with the concept of a fully revealing REE and introduces our approach and the interpretations of the model. Section 4 characterizes the equilibrium and its properties and presents some extensions of the model, the case of inelastic supply and complementarities as well as a robustness exercise on the information structure. Section 5 deals with information acquisition and the GrossmanStiglitz paradox and checks that the results are robust, and Section 6 applies the results to banks bidding for liquidity and the impact of crises. Section 7 considers large but finite markets and the approximation results to the continuum limit. Concluding remarks close the paper and the Appendix gathers the proofs of the results.

\section{The model}

The base model we consider is a continuum version of the finite trader supply competition model of Vives (2011). We posit here that agents compete in demand functions but the difference is immaterial. A continuum of traders-indexed in the unit interval $i \in[0,1]$, which is endowed with the Lebesgue measure-face a linear, downward-sloping inverse supply for a homogenous good $p=\alpha+\beta \tilde{x}$. Here $\alpha, \beta>0$ and $\tilde{x}$ denotes aggregate quantity in our continuum economy (and also per capita quantity, since we have normalized the measure of traders to 1 ). We have $\tilde{x}=\int_{0}^{1} x_{i} d i$, where $x_{i}$ is the individual quantity demanded by trader $i$. We interpret $x_{i}<0$ to mean that the trader is a (net) supplier.

\footnotetext{
12 Rostek and Weretka (2012) consider also competition in schedules and introduce an asymmetric correlation structure for valuation parameters.
} 
Traders are assumed to be risk neutral. The profits of trader $i$ when the price is $p$ are

$$
\pi_{i}=\left(\theta_{i}-p\right) x_{i}-\frac{\lambda}{2} x_{i}^{2}
$$

where $\theta_{i}$ is a value idiosyncratic to the trader and $\lambda x_{i}$ is a marginal transaction, opportunity or limit to arbitrage cost (it could also be interpreted as a proxy for risk aversion).

We assume that $\theta_{i}$ is normally distributed (with mean $\bar{\theta}>\alpha$ and variance $\sigma_{\theta}^{2}$ ). The parameters $\theta_{i}$ and $\theta_{j}, j \neq i$, are correlated with correlation coefficient $\rho \in[0,1]$. We therefore have $\operatorname{cov}\left[\theta_{i}, \theta_{j}\right]=\rho \sigma_{\theta}^{2}$ for $j \neq i$. Trader $i$ receives a signal $s_{i}=\theta_{i}+\varepsilon_{i}$; all signals are of the same precision, and $\varepsilon_{i}$ is normally distributed with $\mathbb{E}\left[\varepsilon_{i}\right]=0$ and $\operatorname{var}\left[\varepsilon_{i}\right]=\sigma_{\varepsilon}^{2}$. Error terms in the signals are correlated neither with themselves nor with the $\theta_{i}$ parameters.

Our information structure encompasses the case of a common value and also that of private values. If $\rho=1$, the valuation parameters are perfectly correlated and we are in a common value model. When $0<\rho<1$, we are in a private values model if signals are perfect and $\sigma_{\varepsilon}^{2}=0$ for all $i$; traders receive idiosyncratic, imperfectly correlated shocks, and each trader observes her shock with no measurement error. If $\rho=0$, then the parameters are independent and we are in an independent values model. Under our assumption of normality, conditional expectations are affine. ${ }^{13}$

13 With Gaussian distributions there is positive probability that prices and quantities are negative in equilibrium. We can control for this if necessary by restricting the variances of the distributions and of the parameters $\alpha, \beta, \lambda$, and $\bar{\theta}$. 
Let the average valuation parameter be $\tilde{\theta} \equiv \int \theta_{j} d j$, normally distributed with mean $\bar{\theta}$ and $\operatorname{cov}\left[\tilde{\theta}, \theta_{i}\right]=\operatorname{var}[\tilde{\theta}]=\rho \sigma_{\theta}^{2} \cdot{ }^{14}$ Note that $\mathbb{E}\left[\theta_{i} \mid \tilde{\theta}\right]=\tilde{\theta}$. The dispersion in valuations is given by $\mathbb{E}\left[\left(\theta_{i}-\tilde{\theta}\right)^{2}\right]=(1-\rho) \sigma_{\theta}^{2}$. It is maximal for $\rho=0$ and minimal for $\rho=1$. An equivalent formulation that highlights the aggregate and idiosyncratic components of uncertainty is to let $\eta_{i} \equiv \theta_{i}-\tilde{\theta}$ and observe that $\theta_{i}=\tilde{\theta}+\eta_{i}$, where $\operatorname{cov}\left[\eta_{i}, \tilde{\theta}\right]=0$ and $\operatorname{cov}\left[\eta_{i}, \eta_{j}\right]=0$ for $i \neq j$. It is worth emphasizing that signal $s_{i}=\tilde{\theta}+\eta_{i}+\varepsilon_{i}$ provides "bundled" information on the aggregate $\tilde{\theta}$ and idiosyncratic $\eta_{i}$ components.

We adopt the convention that the average of independent and identically distributed random variables with mean zero is zero. ${ }^{15}$ We then have $\tilde{s} \equiv \int s_{i} d i=\int \theta_{i} d i+\int \varepsilon_{i} d i=\tilde{\theta}$ almost surely, since $\int \varepsilon_{i} d i=0$ according to our convention. Note that if $\rho=0$ then $\tilde{\theta}=\bar{\theta} \cdot{ }^{16}$

\section{Rational expectations equilibrium and the demand schedule game}

In this section we begin by defining REE and expounding on its problems. We then move on to our game-theoretic approach and interpretations of the model.

14 This can be justified as the continuum analogue of the finite case with $n$ traders. Then, under our assumptions, the average parameter $\tilde{\theta}_{n}$ is normally distributed with mean $\bar{\theta}$, $\operatorname{var}\left[\tilde{\theta}_{n}\right]=(1+(n-1) \rho) \sigma_{\theta}^{2} n^{-1}$, and $\operatorname{cov}\left[\tilde{\theta}_{n}, \theta_{i}\right]=\operatorname{var}\left[\tilde{\theta}_{n}\right]$. The result is obtained by letting $n$ tend to infinity.

15 See Vives (1988) for a justification of this convention. In any event, we will see in Section 7 that the equilibrium in the continuum economy with positive private precision is the limit of equilibria in the appropriate finite economies under the standard laws of large numbers.

16 Equality among random variables has to be understood to hold almost surely (a.s.). We will not insist on this in the paper. 


\subsection{Rational expectations equilibrium}

A (competitive) rational expectations equilibrium is a (measurable) price function mapping the average valuation (state of the world) $\tilde{\theta}$ into prices $P(\tilde{\theta})$ and a set of trades $x_{i}, i \in[0,1]$, such that the following two statements hold.

(1) Trader i maximizes its expected profit, $\mathbb{E}\left[\pi_{i} \mid s_{i}, p\right]$, conditional on knowing the functional relationship $P(\tilde{\theta})$ as well as the underlying distributions of the random variables.

(2) Markets clear: $Z(p) \equiv \int_{0}^{1} x_{i} d i-\beta^{-1}(p-\alpha)=0$.

Thus each trader optimizes while taking prices as given, as in the usual competitive equilibrium, but infers from prices the relevant information.

This equilibrium concept may be problematic. Consider the common value case $(\rho=1)$; we shall present a fully revealing REE that is not implementable. Suppose there is a competitive equilibrium of a full information market in which the traders know $\theta$. At this equilibrium, price equals marginal benefit, $p=\theta-\lambda x_{i}$; therefore, individual demand is $x_{i}=\lambda^{-1}(\theta-p)$. The equilibrium price is given by the market-clearing condition $Z(p)=0$ and is equal to $p=(\lambda \alpha+\beta \theta) /(\lambda+\beta)$. This allocation is also a fully revealing REE of our economy (Grossman (1981)). Indeed, looking at the price allows each trader to learn $\theta$, which is the only relevant uncertainty, and the allocation is a REE equilibrium because traders optimize and markets clear. However, this REE has a strange property: the price is fully revealing even though a trader's demand -easily seen by inverting the price to recover $\theta$ and substituting in the demand $x_{i}=\lambda^{-1}(\mathbb{E}[\theta \mid p]-p)$ to be given by $x_{i}=\beta^{-1}(p-\alpha)$, and therefore equal to per capita supply- is independent of the signals received. The question is then how has the information been incorporated into 
the price or what is the game and the market microstructure that yields such a result. In this case we cannot find a game that delivers as an equilibrium the fully revealing REE. ${ }^{17}$

A similar fully revealing equilibrium can be displayed when traders receive no private signal $\left(\sigma_{\varepsilon}^{2} \rightarrow \infty\right)$ and $0<\rho<1$. Then the equilibrium price in a full information market is $p=(\lambda \alpha+\beta \tilde{\theta}) /(\lambda+\beta)$ and it corresponds also to a (fully revealing) REE in which traders obtain $\tilde{\theta}$ by conditioning on the price. In this equilibrium, using the fact that $\mathbb{E}\left[\theta_{i} \mid \tilde{\theta}\right]=\tilde{\theta}$, we have also that the demand of a trader is equal to per capita supply $x_{i}=\beta^{-1}(p-\alpha)$.

\subsection{The demand schedule game}

We will restrict our attention to REE that are the outcome of a well-specified game - that is, implementable REE. The natural way to implement competitive REE in our context is to consider competition among demand functions (see Wilson (1979); Kyle (1989)) in a market where each trader is negligible. ${ }^{18}$

We assume that traders compete in terms of their demand functions for the exogenous supply of the good. The game's timing is as follows. At $t=0$, random variables $\left(\theta_{i}\right)_{i \in[0,1]}$ are drawn but not observed. At $t=1$, traders observe their own private signals, $\left(s_{i}\right)_{i \in[0,1]}$, and submit demand functions $X_{i}\left(s_{i}, \cdot\right)$ with $x_{i}=X_{i}\left(s_{i}, p\right)$, where $p$ is the market price. The strategy of a trader is therefore a map from the signal space to the space of demand functions. At $t=2$ the market clears, demands are aggregated and

17 If we were to insist that prices be measurable in excess demand functions, then the fully revealing REE would not exist (see Beja (1977); Anderson and Sonnenschein (1982)). However, fully revealing REE are implementable if each agent is informationally "small" or irrelevant in the sense that his private information can be predicted from the joint information of other agents (Palfrey and Srivastava (1986); Postlewaite and Schmeidler (1986); Blume and Easley (1990)).

18 See Gul and Postlewaite (1992) and Mas-Colell and Vives (1993) for results on the implementation of efficient allocations in large economies. 
crossed with supply to obtain an equilibrium price, ${ }^{19}$ and payoffs are collected. An implementable REE is associated with a Bayesian Nash equilibrium of the game in demand functions. Hereafter we discuss only the linear Bayesian demand function equilibrium (DFE).

The model and game admit several interpretations in terms of financial markets and markets for goods as long as there are enough participants to justify the use of the continuum model assumption (this issue is dealt formally with in Section 7). In particular, the good may be a financial asset such as central bank funds or Treasury notes, and the traders are the bidders (banks and other intermediaries) in the auction who use demand functions (in Section 6 we develop the application to liquidity auctions). The good could also be an input (such as labor of uncertain productivity) whose traders are the firms that want to purchase it. ${ }^{20}$

\section{Bayesian demand function equilibrium}

In this section we use Proposition 1 to characterize the symmetric ${ }^{21}$ equilibrium of the demand schedule game before discussing its properties. We then extend the range of the model to double auctions and inelastic supply and market structures with complementarities.

Proposition 1. Let $\rho \in(0,1)$ and $\sigma_{\varepsilon}^{2} / \sigma_{\theta}^{2}<\infty$. Then there is a unique symmetric DFE given by

19 If there is no market-clearing price then assume that the market closes; if there are many marketclearing prices, choose the one that maximizes volume.

20 The model also accommodates the case where firms compete in supply functions to fill an exogenous demand, as in procurement auctions. In this case we assume that $\bar{\theta}<\alpha$, since $\theta_{i}$ is now a cost parameter and typically $x_{i}<0$. For example, $\theta_{i}$ could be a unit ex post pollution or emission penalty to be levied on the firm and about which the producer has some private information. See the discussion in Section 6 of Vives (2011a).

21 The symmetry requirement could be relaxed. Then the (linear and symmetric) equilibrium would be unique in the class of (linear) equilibria with uniformly bounded second moments (equivalently, in the class of equilibria with linear price functional of the type $P(\tilde{\theta}))$. See Claim 1 in Vives (2013) for a proof of a similar statement in a related model. 


$$
X\left(s_{i}, p\right)=\left(\mathbb{E}\left[\theta_{i} \mid s_{i}, p\right]-p\right) \lambda^{-1}=b+a s_{i}-c p
$$

Here

$$
a=\frac{1}{\lambda(1+M)}, \quad b=-\frac{\alpha}{\beta}(1-\lambda a), \quad c=\frac{1}{\beta}(a(\beta+\lambda)-1),
$$

and $M=\sigma_{\varepsilon}^{2} /\left((1-\rho) \sigma_{\theta}^{2}\right)$. Moreover, $a>0$ and $-\beta^{-1}<c \leq a \leq \lambda^{-1}$. Also, $c$ is decreasing in $M$ and $\lambda$ and is increasing in $\beta ; Z^{\prime}=-\left(c+\beta^{-1}\right)<0$; and the equilibrium price is given by

$$
p=\frac{\lambda \alpha+\beta \tilde{\theta}}{\lambda+\beta}
$$

It is worthwhile to highlight two properties of this equilibrium:

1) The equilibrium is privately revealing. The price $p$ reveals the average parameter $\tilde{\theta}$ and, for trader $i$, either pair $\left(s_{i}, p\right)$ or $\left(s_{i}, \tilde{\theta}\right)$ is a sufficient statistic for the joint information in the market $\left(s_{i}, \tilde{s}\right)$ in the estimation of $\theta_{i}$. In particular, at equilibrium we have $\mathbb{E}\left[\theta_{i} \mid s_{i}, p\right]=\mathbb{E}\left[\theta_{i} \mid s_{i}, \tilde{\theta}\right]$. The privately revealing character of the equilibrium, which derives from the "bundled" nature of the signal $s_{i}$ about the common $\tilde{\theta}$ and the idiosyncratic component $\eta_{i}$, implies that the incentives to acquire information are preserved under certain conditions as we shall see in Section 5.

2) The equilibrium is efficient: it is a price-taking equilibrium, the price reveals $\tilde{\theta}$, and traders act with a sufficient statistic for the shared information in the economy. ${ }^{22}$ Indeed, at equilibrium we have that price equals marginal benefit with full (shared) information: $p=\mathbb{E}\left[\theta_{i} \mid s_{i}, \tilde{\theta}\right]-\lambda x_{i}$. This would not be the case if traders had market power, since then a wedge would be introduced between price and marginal benefit

22 A fully revealing REE must be ex post Pareto optimal. The reason is that it can be viewed as the competitive equilibrium of an economy with fully informed agents and so, according to the first welfare theorem, it cannot be improved on by a social planner with access to the pooled information of agents (Grossman (1981)). 
(see Vives (2011a)). Neither would the equilibrium be efficient if price were noisy, since then a trader would not take into account the information externality that her trade has on other traders through the effect on the informativeness of the price (see, e.g., Amador and Weill (2010); Vives (2013)).

Let $\rho \in(0,1)$ and $\sigma_{\varepsilon}^{2} / \sigma_{\theta}^{2}<\infty$. When signals are perfect $\left(\sigma_{\varepsilon}^{2}=0\right.$ and $\left.M \equiv \sigma_{\varepsilon}^{2}\left((1-\rho) \sigma_{\theta}^{2}\right)^{-1}=0\right)$, we have that $a=c=\lambda^{-1}, b=0$, and $x_{i}=\lambda^{-1}\left(\theta_{i}-p\right)$. Then bidders have nothing to learn from prices, and the equilibrium is just the usual complete information competitive equilibrium (which, we remark, is independent of $\rho$ ). When $M>0$, bidders learn from prices and the demand functions are steeper: $c<\lambda^{-1}$. Indeed, the larger is $M$ (which is increasing in $\rho$ and in $\sigma_{\varepsilon}^{2} / \sigma_{\theta}^{2}$ ), the more important is the common value component and the steeper are the demand functions (lower $c$ ). The response to a price increase is to reduce the amount demanded according to the usual scarcity effect, but this impulse is moderated (or even reversed) by an information effect, via $\mathbb{E}\left[\theta_{i} \mid s_{i}, p\right]$, because a high price conveys the good news that the average valuation is high (this is akin to adverse selection). Indeed, if $\beta \lambda^{-1}=M$ then $c=0$; for larger values of $M$, we have $c<0$ and demand is upward sloping. Increases in $M$ make the demand function (evaluated at $s_{i}=\bar{\theta}$ ) rotate around the point $(\bar{x}, \bar{p})$ where $\bar{x} \equiv(\alpha-\bar{\theta}) /(\beta+\lambda)$ and $\bar{p} \equiv(\lambda \alpha+\beta \bar{\theta}) /(\beta+\lambda)$ are, respectively, the expected output and price. (See Figure 1.) This follows easily from the fact that $X(\bar{\theta}, p)=\bar{X}-c(p-\bar{p})$. 


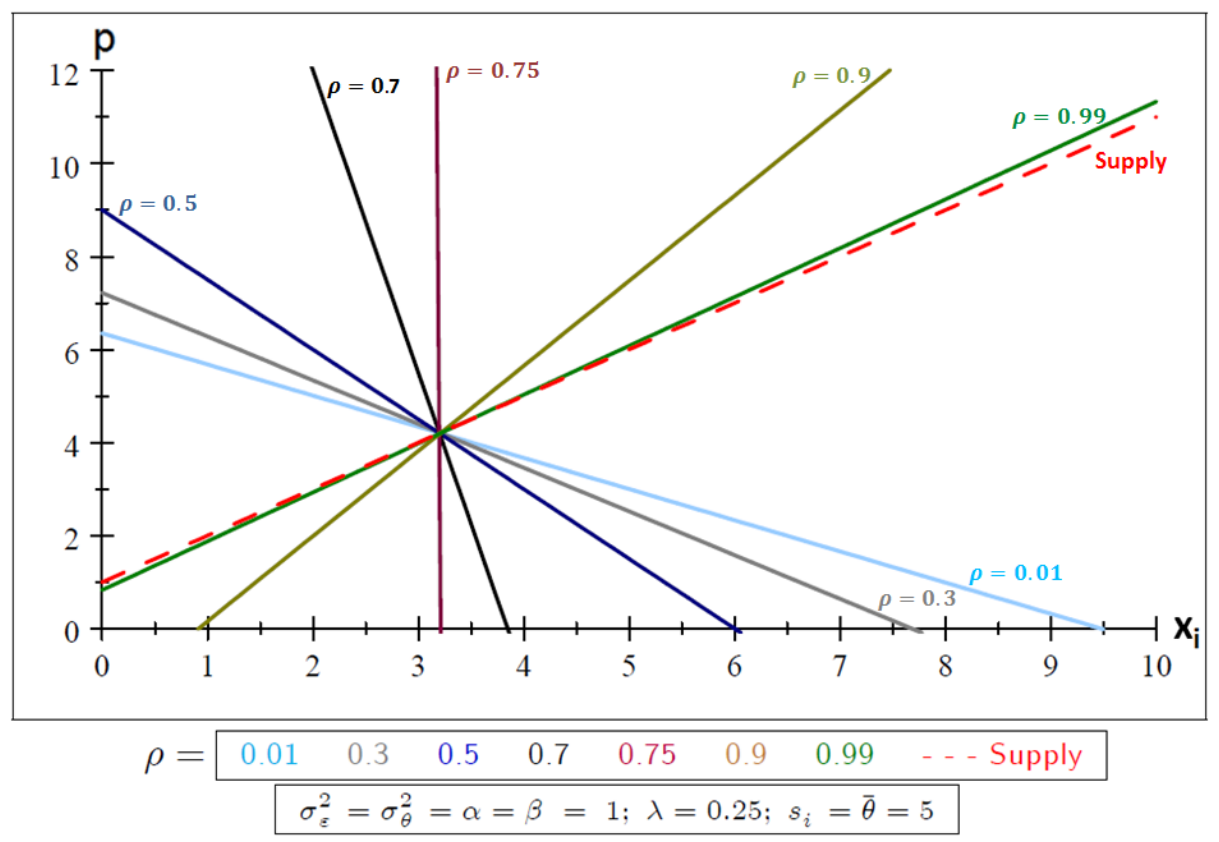

Figure 1. Equilibrium demand as a function of $\rho$. Demands go through the point $(\bar{x}, \bar{p})=(3.2,4.2)$.

As $M \rightarrow \infty$ (be it because $\rho \rightarrow 1$ or $\sigma_{\varepsilon}^{2} \rightarrow \infty$ ) we have that $a \rightarrow 0, b \rightarrow-\alpha / \beta$, and $c \rightarrow-1 / \beta$ and the demand of a trader converges to per capita supply $x_{i}=\beta^{-1}(p-\alpha)$ (as illustrated in Figure 1). Then the linear equilibrium collapses because, in the limit, traders put no weight on their private signals. If $\rho=1$ (and $\left.0<\sigma_{\varepsilon}^{2}<\infty\right)$ then, as stated in Section 3, there is a fully revealing REE but it is not implementable. In the common value case, the equilibrium breaks down. When signals are pure noise $\left(\sigma_{\varepsilon}^{2}=\infty\right)$, the equilibrium is $X(p)=\lambda^{-1}(\bar{\theta}-p)$ because $\mathbb{E}\left[\theta_{i} \mid s_{i}, p\right]=\bar{\theta}$ (even if $\rho=1)$. However, this equilibrium is not the limit of DFE as $\sigma_{\varepsilon}^{2} \rightarrow \infty(M \rightarrow \infty)$. Indeed, both either when $\rho \rightarrow 1$ or $\sigma_{\varepsilon}^{2} \rightarrow \infty$ we have that the limit of DFE is precisely the (non-implementable) fully revealing REE given in Section 3.

If $\rho=0$ then there is asymmetric information but the price conveys no information on values, $c=\lambda^{-1}$, and $X\left(s_{i}, p\right)=\lambda^{-1}\left(\mathbb{E}\left[\theta_{i} \mid s_{i}\right]-p\right)$. Again this is not the 
limit of DFE as $\rho \rightarrow 0$. However, it can be checked that there is no discontinuity in outcomes.

As the transaction cost $\lambda$ increases, $a$ decreases and demand becomes steeper ( $c$ decreases). As $\lambda \rightarrow 0$ and limits to arbitrage disappear we have that $a, c \rightarrow \infty$ and $p$ tends to $\tilde{\theta}$. As $\beta$ decreases supply becomes flatter and equilibrium demand is less elastic until the point it becomes vertical (from $\beta=\lambda M$ ); beyond that point demand is upward sloping, and as $\beta \rightarrow 0$ demand becomes flat at the level $p=\alpha .{ }^{23}$

In summary, demand schedules, as long as they are downward sloping, are steeper with the correlation of valuations and with the noise in the signals; with a steeper slope of the marginal valuation; and with a flatter slope of exogenous supply.

The model can be extended to consider inelastic supply and double auctions, complementarities and more general information structures.

Inelastic supply and double auctions. The case in which an auctioneer supplies $q$ units of the good is easily accommodated by letting $\beta \rightarrow \infty$ and $\alpha / \beta \rightarrow-q$. From the inverse supply function we obtain the average quantity $y=(p-\alpha) / \beta \rightarrow q$; then $c \rightarrow a$ and $b \rightarrow(1-\lambda a) q$. Here demand is always downward sloping, and the strategy of a trader is $X\left(s_{i}, p\right)=(1-\lambda a) q+a\left(s_{i}-p\right)$ and $p=\tilde{\theta}-\lambda q$. The good can be in zero net supply $(q=0)$ as in a double auction, in which case $b=0$ and $p=\tilde{\theta} \cdot{ }^{24} \mathrm{~A}$ trader is a buyer or a seller depending on whether her private signal is larger or smaller than the price. Reny and Perry (2006) obtain a related result in a double auction with a unit mass of traders, each of whom desires at most one unit of the good. In their model there is a common value for the

23 Note that the equilibrium demand can also be written as follows: $X\left(s_{i}, p\right)=(1-\lambda a) \beta^{-1}(p-\alpha)+a\left(s_{i}-p\right)$.

24 In this case there is also a no-trade equilibrium. 
good and the payoff of a trader depends directly on the signal he receives, which provides a private value component to the trader's valuation..$^{25}$

Complementarities. Letting $\beta<0$ allows for complementarities. For example, if traders are suppliers $\left(x_{i}<0\right)$ then $\beta<0$ means that increasing the aggregate quantity leads to price increases, a dynamic typical of network goods; conversely, if traders are demanders $\left(x_{i}>0\right)$ then $\beta<0$ means that increasing the aggregate quantity lowers the price, as may occur with labor supply when the income effect dominates. We can allow negative values of $\beta$ with $0>\beta>-\lambda \alpha / \bar{\theta}$. The last inequality ensures that $\mathbb{E}[p]>0$ in equilibrium and implies that $\beta+\lambda>0$ (since $\bar{\theta}-\alpha>0$ ). When $\beta<0$ we have that $-\beta^{-1}>c \geq a$ and that $c$ increases in $M$; in other words, the exogenous supply is downward sloping and an increase in $M$ makes demand flatter. Furthermore, excess demand is upward sloping: $Z^{\prime}=-\left(c+\beta^{-1}\right)>0$. Now the information and the scarcity effect work in the same direction, and a high price conveys the unequivocal bad news that the average valuation is low.

Generalizing the information structure. Consider the following variation of the information structure where trader $i$ receives the signal $s_{i}=\eta_{i}+\gamma \tilde{\theta}+\varepsilon_{i}=\theta_{i}-(1-\gamma) \tilde{\theta}+\varepsilon_{i}$ (recall that $\left.\eta_{i}=\theta_{i}-\tilde{\theta}\right)$ where $\gamma$ is a real number. We have considered so far the case $\gamma=1$ but outcomes (in terms of prices and quantities) in equilibrium are invariant to the choice of $\gamma \neq 0$ (the price reveals $\tilde{s}=\gamma \tilde{\theta}$ since $\int \eta_{i} d i=0$ and therefore $\tilde{\theta}$ as before). To observe $\left(\theta_{i}-(1-\gamma) \tilde{\theta}+\varepsilon_{i}, \tilde{\theta}\right)$ or $\left(\theta_{i}+\varepsilon_{i}, \tilde{\theta}\right)$ is equivalent. However, the equilibrium demand function changes with $\gamma$. This is so since changing $\gamma$ changes the way prices are used to filter $\tilde{s}$. It is easy to see that the slope of

25 Unlike the case for our DFE, traders in a double action with unit bids cannot condition on the market price because they submit a single bid that is contingent only on private information. Nonetheless, there is a unique (and privately revealing) REE that is implementable as a Bayesian equilibrium of the double auction in symmetric increasing bidding strategies. 
demand is given by $c=\left(\gamma \lambda^{-1}-\beta^{-1} M\right) /(\gamma+M)$. It is worth noting that as $\gamma \rightarrow 0$ we have that $c \rightarrow-1 / \beta$ (slope of per capita supply) and that as $\gamma \rightarrow \infty$ we have that $c \rightarrow 1 / \lambda$ (complete information demand slope).

More in general, Bergemann et al. (2014) show that REE outcomes in the context of the model analyzed and with a general normal information structure can be decentralized with a canonical one-dimensional signal information structure of the form $s_{i}=\eta_{i}+\gamma \tilde{\theta}+\varepsilon_{i}$ (recall that $\eta_{i}=\theta_{i}-\tilde{\theta}$ ) where $\gamma$ is a real number and $\operatorname{cov}\left[\varepsilon_{i}, \varepsilon_{j}\right]=\rho_{\varepsilon} \sigma_{\varepsilon}^{2}$ for $j \neq i$ where $\rho_{\varepsilon} \in[0,1]$. In this context the price reveals the average signal $\tilde{s}=\gamma \tilde{\theta}+\tilde{\varepsilon}$, with $\int \varepsilon_{i} d i=\tilde{\varepsilon} \sim N\left(0, \rho_{\varepsilon} \sigma_{\varepsilon}^{2}\right)$. Therefore, the equilibrium is privately revealing since the trader will use both $s_{i}=\eta_{i}+\gamma \tilde{\theta}+\tilde{\varepsilon}+\left(\varepsilon_{i}-\tilde{\varepsilon}\right)$ and $\tilde{s}=\gamma \tilde{\theta}+\tilde{\varepsilon}$. In the following section we will use this framework to check the robustness of our information acquisition results.

The general information structure considered encompasses also information structures where traders are exposed to an aggregate risk factor (as in Manzano and Vives (2011), who consider a different payoff structure) and prices are not revealing of the fundamental value. Consider the case where we have $\theta_{i}=v+u_{i}$ with $v$ the fundamental value of the asset and $u_{i}=u+\delta_{i}$ a liquidity shock with common component (or aggregate risk) $u$. Trader $i$ knows $u_{i}$ and receives a signal $v+\vartheta_{i}$. (The variables $\delta_{i}$ and $\vartheta_{i}$ are independently distributed, both pairwise and from the rest of random variables.) At a linear equilibrium in this model (under the usual normality assumptions) the price would reveal a linear combination of $v$ and $u$. The equilibrium outcomes can be replicated with the canonical information structure by calibrating appropriately the variance-covariance matrix of the random variables. 


\section{Information acquisition}

Now suppose that private signals must be purchased at a cost, which is increasing and convex in the precision $\tau_{\varepsilon} \equiv 1 / \sigma_{\varepsilon}^{2}$ of the signal, ${ }^{26}$ according to a smooth function $H(\cdot)$ that satisfies $H(0)=0$ with $H^{\prime}>0$ for $\tau_{\varepsilon}>0$, and $H^{\prime \prime} \geq 0$. Hence there are nonincreasing returns to information acquisition. Traders receive signals according to the precision purchased and compete in demand functions. We analyze symmetric Nash equilibria of the covert information acquisition game (one-shot game where traders choose simultaneously the demand function and the precision of the signal). ${ }^{27}$

Suppose that all traders but $i$ have chosen a precision $\tau_{\varepsilon}>0$. Then the market equilibrium (which is unaffected by the actions of a single trader) exhibits, according to Proposition 1 , the price $p=(\lambda \alpha+\beta \tilde{\theta}) /(\lambda+\beta)$, a price that reveals $\tilde{\theta}$. This implies that the expected profit (gross of the information cost) of trader $i$ is given by $\mathbb{E}\left[\pi_{i}\right]=\frac{\lambda}{2} \mathbb{E}\left[x_{i}^{2}\right]$, where $x_{i}=\left(\mathbb{E}\left[\theta_{i} \mid s_{i}, \tilde{\theta}\right]-p\right) / \lambda$. It does not depend on the average precision $\tau_{\varepsilon}$, because the price reveals $\tilde{\theta}$, but it does depend on his precision of information $\tau_{\varepsilon_{i}}$ since his private signal is used to estimate $\theta_{i}$. It can be checked then that the marginal benefit of increasing $\tau_{\varepsilon_{i}}$ is

$$
\frac{\partial \mathbb{E}\left[\pi_{i}\right]}{\partial \tau_{\varepsilon_{i}}}=\frac{(1-\rho)^{2}}{2 \lambda\left(\tau_{\theta}+(1-\rho) \tau_{\varepsilon_{i}}\right)^{2}} .
$$

This marginal benefit is decreasing in $\rho, \tau_{\varepsilon_{i}}, \tau_{\theta}$ and $\lambda$ provided that $\rho<1$. This fact leads to the following result.

26 For a random variable $\eta$, we use $\tau_{\eta}$ to denote $1 / \sigma_{\eta}^{2}$. We argue for a symmetric equilibrium, but in fact, it can be shown that neither is there an asymmetric equilibrium in the class of trading strategies with bounded second moments.

27 We argue for a symmetric equilibrium, but in fact, it can be shown that neither is there an asymmetric equilibrium in the class of trading strategies with bounded second moments. 
Proposition 2. Let $\rho \in[0,1]$ and $\bar{\rho} \equiv 1-\tau_{\theta} \sqrt{2 \lambda H^{\prime}(0)}$. We have that:

(i) If $\left(2 \lambda \tau_{\theta}^{2}\right)^{-1} \leq H^{\prime}(0)$, or equivalently $\bar{\rho} \leq 0$, then $\tau_{\varepsilon}^{*}=0$ is the unique symmetric equilibrium.

(ii) If $H^{\prime}(0)<(1-\rho)^{2}\left(2 \lambda \tau_{\theta}^{2}\right)^{-1}$, or equivalently $\bar{\rho}>\rho \geq 0$, then $\tau_{\varepsilon}^{*}>0$ is the unique symmetric equilibrium, and $\tau_{\varepsilon}^{*}$ is decreasing in $\lambda, \tau_{\theta}$, and $\rho$.

(iii) If $\left(2 \lambda \tau_{\theta}^{2}\right)^{-1}>H^{\prime}(0) \geq(1-\rho)^{2}\left(2 \lambda \tau_{\theta}^{2}\right)^{-1}$, or equivalently $\rho \geq \bar{\rho}>0$, there is no equilibrium.

In short, if $\bar{\rho} \leq 0$ there is no information acquisition since the marginal benefit of acquiring information at 0 is lower than the marginal $\operatorname{cost}\left(2 \lambda \tau_{\theta}^{2}\right)^{-1} \leq H^{\prime}(0)$. If $\bar{\rho}>0$ $\left(\left(2 \lambda \tau_{\theta}^{2}\right)^{-1}>H^{\prime}(0)\right)$ then with not too high correlation, $\rho<\bar{\rho}$, there is an equilibrium with information acquisition. However, as $\rho \rightarrow \bar{\rho}$ we have $\tau_{\varepsilon}^{*} \rightarrow 0$, and the demand function equilibrium collapses. With high correlation, $\rho \geq \bar{\rho}>0$, we would have no information acquisition at a candidate equilibrium but in fact no equilibrium exists.

Indeed, if $\left(2 \lambda \tau_{\theta}^{2}\right)^{-1}>H^{\prime}(0)$ it will benefit a single trader to purchase information if the others do not and no information acquisition cannot be an equilibrium. (See Figure 2.)

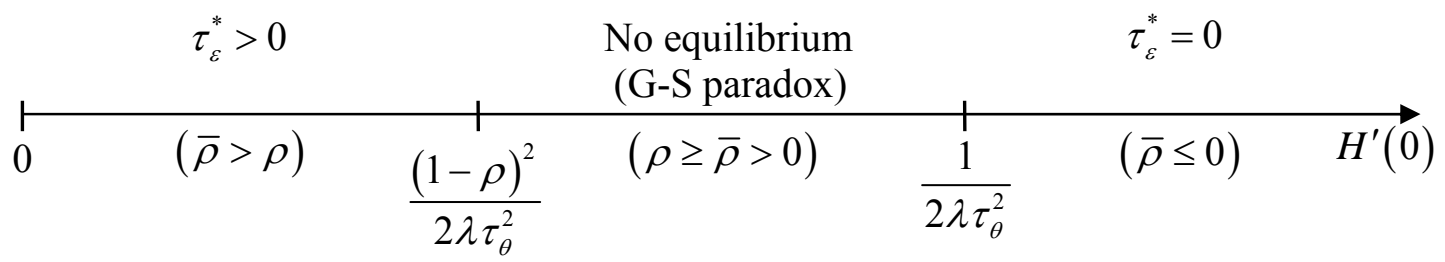

Figure 2. The range of the Grossman-Stiglitz paradox in the information acquisition game (where: $\bar{\rho}=1-\tau_{\theta} \sqrt{2 \lambda H^{\prime}(0)}$ ). 
The range of parameters where there is no equilibrium, $\rho \geq 1-\tau_{\theta} \sqrt{2 \lambda H^{\prime}(0)}>0$, is where the Grossman-Stiglitz paradox of the impossibility of an informationally efficient market survives. Therefore, in particular, we find that when $\rho<1$ an equilibrium exists if $H^{\prime}(0)=0$ or if the prior is diffuse ( $\tau_{\theta}$ small). In this case moving away from the pure common value case we resolve the Grossman-Stiglitz paradox. In general, a more diffuse prior, a lower cost of transacting $\lambda$, or less correlation of valuations induces more acquisition of information. In fact, as $\lambda \rightarrow 0$ we have that $\tau_{\varepsilon}^{*} \rightarrow \infty$. Hence we see that the incentives to acquire information are preserved because the equilibrium is privately revealing - as long as we are not too close to the common value case, or otherwise the marginal cost of acquiring information at zero precision is zero (and $\rho<1) .28$

It is worth noting that in contrast to the classical model with noise traders where there is an upper bound in price informativeness (e.g. Verrecchia (1982)) here either the price is privately revealing or not at all. In the classical case information acquisition decisions are strategic substitutes and the more informative is the price, the less incentives there are to acquire information. In fact, the result in the Grossman-Stiglitz model is that price informativeness is constant as noise trading vanishes. In our model information acquisition decisions are strategically independent since once a positive mass of traders buy information the price is fully revealing of $\tilde{\theta}$.

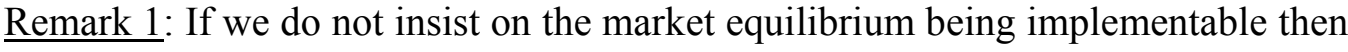
equilibrium exists even in the region $\left(2 \lambda \tau_{\theta}^{2}\right)^{-1}>H^{\prime}(0) \geq(1-\rho)^{2}\left(2 \lambda \tau_{\theta}^{2}\right)^{-1}$. Indeed, recall that (as discussed in Section 3.1) when $\tau_{\varepsilon}=0$ there is a fully revealing equilibrium with

28 When $\rho<1$ the same outcome would obtain as a subgame-perfect equilibria of the two-stage game where traders choose first the precision of information, which average is observable, and then compete in demand functions contingent on the signals received. Equivalence of the games follows from the existence of a continuum of traders. When $\rho=1$ in the sequential game if at the first stage traders were to choose a positive average precision then no equilibrium could exist at the market stage. 
price $p=(\lambda \alpha+\beta \tilde{\theta}) /(\lambda+\beta)$. In the parameter region described above, $\tau_{\varepsilon}^{*}=0$ is an equilibrium with endogenous information acquisition. This is so since if no trader acquires information, then a single trader learns $\tilde{\theta}$ from the price and since $H^{\prime}(0) \geq(1-\rho)^{2}\left(2 \lambda \tau_{\theta}^{2}\right)^{-1}$ has no incentive to acquire information either.

Welfare analysis. It can be shown (see Lemma $\mathrm{A}$ in the Appendix) that for $H^{\prime}(0)<(1-\rho)^{2}\left(2 \lambda \tau_{\theta}^{2}\right)^{-1}$ the private and social incentives to purchase information are aligned: the marginal social and private benefits are the same and the market acquires the right amount of information $\tau_{\varepsilon}^{*}>0$. However, when $H^{\prime}(0) \geq(1-\rho)^{2}\left(2 \lambda \tau_{\theta}^{2}\right)^{-1}$ and $\rho>0$ there is no welfare-optimal level of information purchase because of a discontinuity at $\tau_{\varepsilon}=0$ : We have that expected gross total surplus $\mathbb{E}[\mathrm{TS}]$ is decreasing in $\tau_{\varepsilon}$ for $\tau_{\varepsilon}>0$ but $\lim _{\tau_{\varepsilon} \rightarrow 0^{+}} \mathbb{E}[\mathrm{TS}]=\frac{1}{2} \frac{(\bar{\theta}-\alpha)^{2}+\rho \sigma_{\theta}^{2}}{\beta+\lambda}$ while $\left.\mathbb{E}[\mathrm{TS}]\right|_{\tau_{\varepsilon}=0}=\frac{1}{2} \frac{(\bar{\theta}-\alpha)^{2}}{\beta+\lambda}$ is strictly lower for $\rho>0$. The planner would like to set $\tau_{\varepsilon}$ as low as possible but $\tau_{\varepsilon}=0$ delivers less surplus. The discontinuity arises since the price is revealing of $\tilde{\theta}$ when $\tau_{\varepsilon}>0$ but not when $\tau_{\varepsilon}=0$ for $\rho>0$.

Robust information structures. The Grossman-Stiglitz paradox can be resolved despite the price being fully revealing of the average parameter $\tilde{\theta}$ because the signal $S_{i}$ provides "bundled" information on the common $\tilde{\theta}$ and the idiosyncratic $\eta_{i}$ components of the valuation of a trader. This result is robust to general information structures as long as the bundled character of the signal is present.

Consider the following version of the canonical one-dimensional information structure in Bergemann et al. (2014) with $s_{i}=\eta_{i}+\gamma \tilde{\theta}+\omega+\varepsilon_{i}=\theta_{i}-(1-\gamma) \tilde{\theta}+\omega+\varepsilon_{i}$ where $\gamma$ is a real number, representing the direct strength of the common fundamental component in the signal, and $\omega$ is a common noise component in the signal, normally 
distributed (with mean 0 and variance $\sigma_{\omega}^{2} \geq 0$ and independent of the rest of random variables in the model), and the rest of the model is as in Section 2. Note that the signal increases with both $\theta_{i}$ and $\tilde{\theta}$ only if $\gamma>1$. In this context the price reveals $\tilde{s}=\gamma \tilde{\theta}+\omega$, provided that $\tau_{\varepsilon}>0$, and therefore $\mathbb{E}\left[\theta_{i} \mid s_{i}, p\right]=\mathbb{E}\left[\theta_{i} \mid \hat{s}_{i}, \tilde{s}\right]$ where $\hat{s}_{i}=\eta_{i}+\varepsilon_{i}$. It is not difficult to check that the marginal benefit of increasing $\tau_{\varepsilon_{i}}$ for trader $i$ is exactly as before whenever the other traders have acquired positive precision. It follows that there is an equilibrium with positive information acquisition in the same circumstances (and with the same level of precision) as in Proposition 2. The reason is that $\operatorname{cov}\left[\hat{s}_{i}, \tilde{s}\right]=0$ and therefore the parameters $\sigma_{\omega}^{2}$ and $\gamma$ do not influence the marginal profitability of changing $\tau_{\varepsilon_{i}}$. Furthermore, it can be checked also that the marginal benefit of increasing $\tau_{\varepsilon_{i}}$ when the other traders do not purchase the signal, evaluated at $\tau_{\varepsilon_{i}}=0$, is given by $(1+\rho(\gamma-1))\left(2 \lambda \tau_{\theta}^{2}\right)^{-1}$. Note that this, indeed, coincides with the original model if $\gamma=1$ or $\rho=0$. It follows that Proposition 2 can be generalized to the canonical information structure $s_{i}=\eta_{i}+\gamma \tilde{\theta}+\omega+\varepsilon_{i}$ as follows: (i) $\tau_{\varepsilon}^{*}=0$ if $(1+\rho(\gamma-1))^{2}\left(2 \lambda \tau_{\theta}^{2}\right)^{-1} \leq H^{\prime}(0)$; (ii) holds exactly; and (iii) there is no equilibrium if $(1+\rho(\gamma-1))^{2}\left(2 \lambda \tau_{\theta}^{2}\right)^{-1}>H^{\prime}(0) \geq(1-\rho)^{2}\left(2 \lambda \tau_{\theta}^{2}\right)^{-1}$. This region exists if and only if $\rho \gamma>0$, and it grows with $\gamma$, and with $\rho$ if $\gamma \geq 1$. (See Proposition $2 *$ in the Appendix for the complete statement and proof.)

If $\gamma=1$ we recover, indeed, Proposition 2 (which, incidentally, holds for any value of $\left.\tau_{\omega}\right)$. Note that if $\gamma=0$ or $\rho=0$ then $(1+\rho(\gamma-1))^{2}\left(2 \lambda \tau_{\theta}^{2}\right)^{-1}=(1-\rho)^{2}\left(2 \lambda \tau_{\theta}^{2}\right)^{-1}$ (indeed, in this case the signal is purely idiosyncratic and it does not matter whether other traders purchase information or not). The length of the non-existence of equilibrium interval is increasing with the strength of the common fundamental component in the signal (which grows with $\gamma$, and also with $\rho$ if $\gamma \geq 1$ ). When $\gamma<0$ then equilibrium 
always exists and in the region $(1+\rho(\gamma-1))^{2}\left(2 \lambda \tau_{\theta}^{2}\right)^{-1} \leq H^{\prime}(0)<(1-\rho)^{2}\left(2 \lambda \tau_{\theta}^{2}\right)^{-1}$ there are multiple equilibria, one with and another without information acquisition.

In summary, for $0<\rho<1$ we have that the signal $s_{i}$ provides "bundled" information on the common $\tilde{\theta}$ and the idiosyncratic $\eta_{i}$ components of the valuation of a trader and there is equilibrium except if $H^{\prime}(0)$ is in an intermediate region which is increasing with the strength of the common component in the signal, represented by increases in $\gamma$ and in $\rho$ when $\gamma \geq 1$.

\section{Banks bidding for liquidity and the crisis.}

In this section we consider the case of banks bidding for liquidity and the impact of a crisis. ${ }^{29}$ The good is a financial asset such as central bank funds and the traders are the bidders (banks and other intermediaries) in the liquidity auction who use demand functions. In the open-market operation of central bank funds, the average valuation $\tilde{\theta}$ is related to the average price (interest rate) in the secondary interbank market, which is mostly over-the-counter. The idiosyncratic valuation $\theta_{i}$ for bank $i$ reflects thus the terms that this bank obtains in the secondary market as well as its liquidity needs (because of reserve requirements for example) and the bank receives an imperfect signal about its valuation. A bidder bank must offer the central bank collateral in exchange for funds, and the bidder's first preference is to offer the least liquid one. Given an increased allotment of funds, the bank must offer more liquid types of collateral at a higher opportunity cost; this implies a declining marginal valuation for the bidder with $\lambda$ reflecting the structure of a counterparty's pool of collateral. ${ }^{30}$ There is evidence also of the winner's curse in liquidity auctions indicating a common value component in the demands of banks (this is the case for the longer term refinancing operations (LTROs) of the ECB, where the number of bidders is large, see Linzert et al. (2007)).

\footnotetext{
29 See also the related discussion in Vives (2010).

30 See Ewerhart et al. (2010) and Cassola et al. (2013).
} 


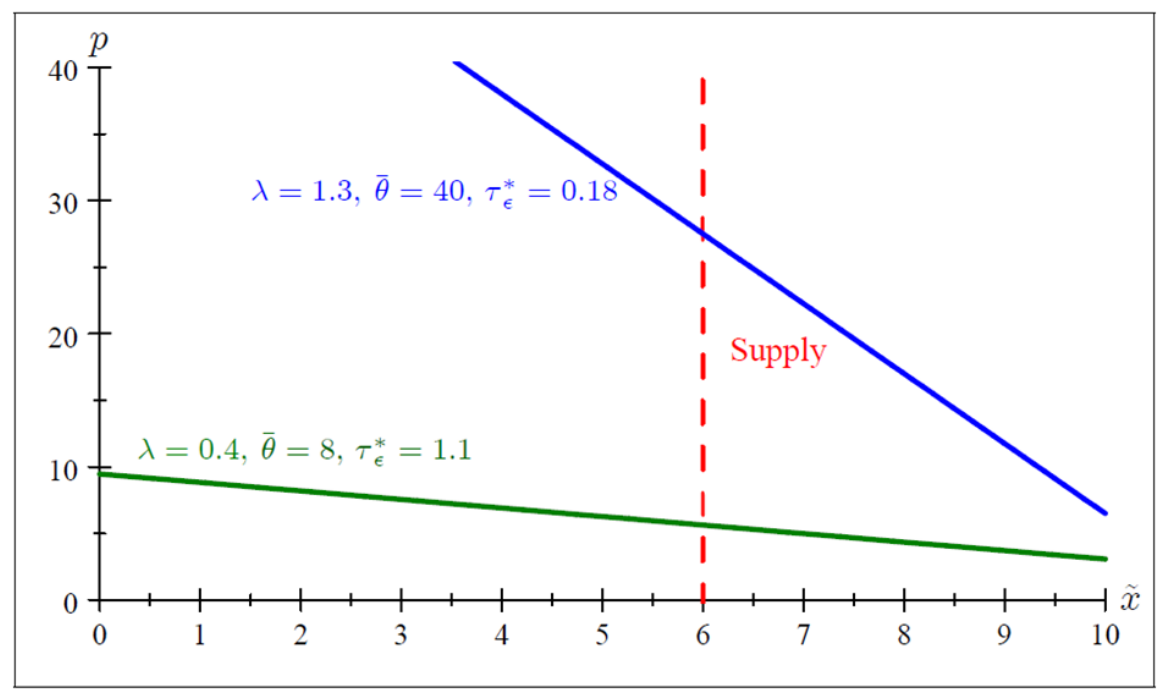

$\rho=0.5 ; \tau_{\theta}=0.33 ; q=6 ; \gamma_{1}=0.2 ; \gamma_{2}=0.15$

Figure 3. Impact of a crisis. Equilibrium demands with inelastic supply and endogenous precision when $\lambda$ and $\bar{\theta}$ increase and, as a consequence, equilibrium precision $\tau_{\varepsilon}^{*}$ decreases where $H\left(\tau_{\varepsilon}\right)=\gamma_{1} \tau_{\varepsilon}+\left(\gamma_{2} / 2\right) \tau_{\varepsilon}^{2}$.

In this scenario we may expect that the correlation $\rho$ of the values of the banks increases (equivalently, that the volatility of the average price $\tilde{\theta}$ in the secondary market for liquidity increases) and that $\lambda$ also increases as it becomes more costly to supply more liquid collateral (this may correspond to a decrease in collateral quality). ${ }^{31}$ The direct effect of an increase in $\rho$ or $\lambda$ is to make the demand schedules of the banks steeper (Proposition 1), and this effect is reinforced by the induced decrease in information precision ( $\tau_{\varepsilon}^{*}$ goes down, according to Proposition 2). The effect of the crisis is thus that demand schedules are steeper and the signals noisier. (See Figure 3 where the model with inelastic supply is simulated.) These effects are consistent with the empirical evidence gathered by Cassola et al (2013) when studying European Central Bank auctions and by Allen, Hortaçsu, and Kastl (2011) for Canadian liquidity auctions. These authors find, respectively, that the aggregate bid curve became steeper after the subprime

31 See Heider, Hoerova and Holthausen (2009). 
crisis in August 2007 and after Lehman's turmoil in 2008. ${ }^{32}$ (See Figure 4, taken from Allen et al. (2011).)

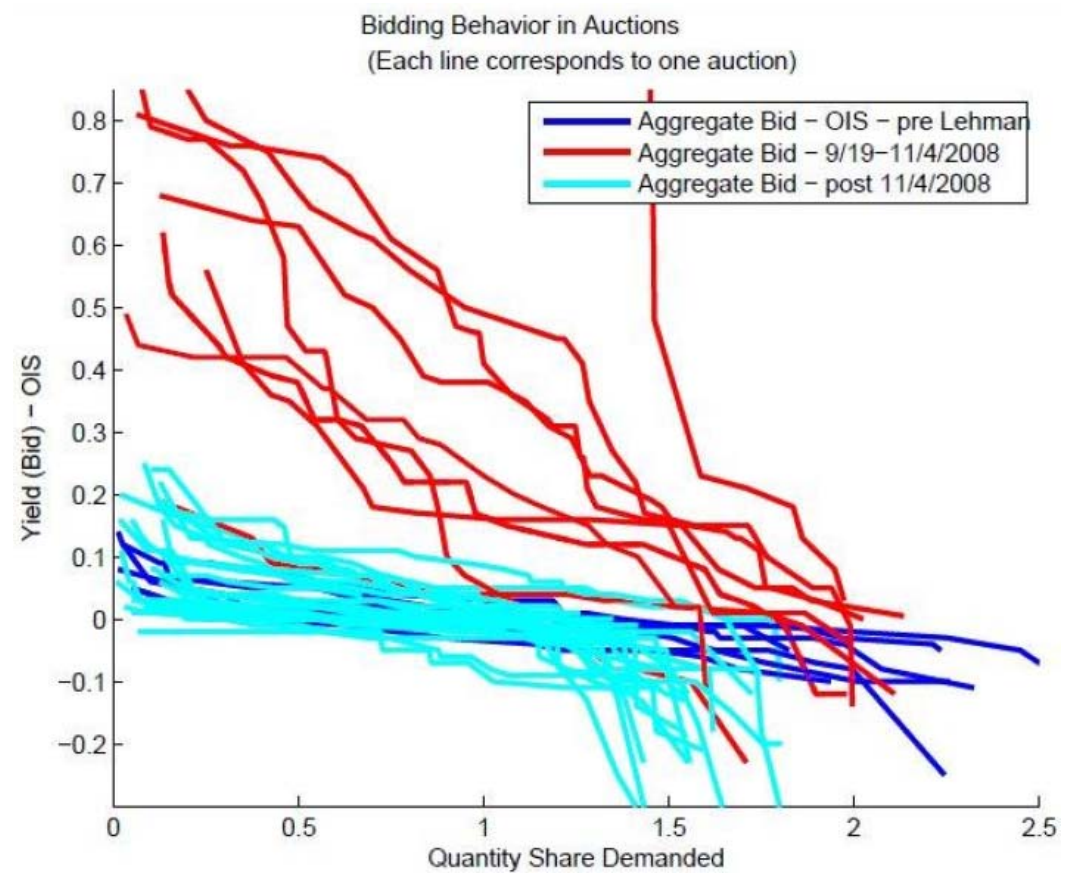

Figure 4. Aggregate demand curves in Canadian liquidity auctions (Fig. 4 in Allen et al. (2011)) and how they become steeper after the crisis of Lehman Brothers and go back to normal afterwards. The vertical axis displays the bid minus the Overnight Index Swap (OIS). ${ }^{33}$

\section{Finite markets and convergence to the limit equilibrium}

The question arises of whether the results obtained in the large market are simply an artifact of the continuum specification. In this section, we answer this question in the negative whenever the equilibrium calls for some information acquisition. In this case, we show, building on the results in Vives (2011), that the equilibria in finite markets tend to the equilibrium of the continuum economy as the market grows large, which justifies our use of a continuum model to approximate the large market. Furthermore, we check how large a market has to be for the continuum approximation to be useful by computing

32 Market power leading to bid shading may reinforce the steepness of the bid curve (see Vives (2011a)). It should be noted, however, that both ECB and Canadian auctions have been discriminatory, and not uniform price, so far.

33 The spread measures the difference between bids and the rates paid on overnight index swaps (OIS), instruments that are not exposed to the default risk of intermediaries. 
the rate of convergence to the continuum limit. We also check in what cases the equilibrium in the continuum economy is not a good approximation of large finite markets.

\subsection{Equilibrium in a finite market and convergence}

Consider the following replica economy. Suppose that inverse supply is given by $P_{n}(y)=\alpha+\beta n^{-1} y$; here $y$ is total quantity and $n$ is the number of traders (buyers), each with the same benefit function as before. Increasing $n$ will increase the number of buyers and increase the supply at the same rate. Denote with subscript $n$ the magnitudes in the $n$-replica market. The information structure is the finite-trader counterpart of the structure described in Section 2. We have that $\tilde{\theta}_{n} \equiv\left(\sum_{i=1}^{n} \theta_{i}\right) / n \sim N\left(\bar{\theta},(1+(n-1) \rho) n^{-1} \sigma_{\theta}^{2}\right)$ and $\operatorname{cov}\left[\tilde{\theta}_{n}, \theta_{i}\right]=\operatorname{var}\left[\tilde{\theta}_{n}\right]$. As $n$ grows large the finite economy converges to the continuum economy since $n^{-1} y$ is average quantity and $\tilde{\theta}_{n} \rightarrow \tilde{\theta} \sim N\left(\bar{\theta}, \rho \sigma_{\theta}^{2}\right)$ in mean square, and that $\operatorname{cov}\left[\tilde{\theta}_{n}, \tilde{\theta}\right]=\operatorname{var}[\tilde{\theta}] .{ }^{34}$ The following Lemma characterizes the equilibrium in the finite market.

Lemma 1 (Proposition 1, Vives (2011a)): In the $n$-replica market there is a unique (symmetric) DFE for $\rho<1$. At equilibrium

$$
X_{n}\left(s_{i}, p\right)=\left(\mathbb{E}\left[\theta_{i} \mid s_{i}, p\right]-p\right)\left(d_{n}+\lambda\right)^{-1}=b_{n}+a_{n} s_{i}-c_{n} p
$$

where $d_{n}=\left(\beta^{-1} n+(n-1) c_{n}\right)^{-1}>0$.

The equilibrium is privately revealing, and the price reveals the average signal of the traders, $\tilde{s}_{n}$. The equilibrium parameter $d_{n}$ is the slope of the residual supply that a trader faces in equilibrium and it is increasing in $\rho$. That is, $d_{n}$ is the wedge or distortion introduced by market power in the presence of asymmetric information in the finite market. In a price-taking equilibrium, such as in the continuum market, we have $d=0$.

34 See the Appendix for definitions of "in mean square" and of convergence (and rates of convergence) for random variables. 
As $\rho \rightarrow 1, c_{n} \rightarrow-1 / \beta$ and $d_{n} \rightarrow \beta$, and we approach the collusive outcome. With symmetric information (either $\tau_{\varepsilon}=0$ or $\tau_{\varepsilon}=\infty$ ) the equilibrium is independent of $\rho$, it exists even if $\rho=1$, and $d_{n}$ equals the full information equilibrium level market power distortion $d_{n}^{f}$. This level of market power is also attained if $\rho=0$ since then there is nothing to learn from prices.

In a finite $n$-replica market, traders have the capacity to influence prices; and the price reveals the average signal of the traders $\tilde{s}_{n}$, which is a noisy estimate of $\tilde{\theta}_{n}$. From Vives (2011a) we know that convergence to price-taking behavior as the market grows is at the rate $1 / n$, since the market power index $d_{n}$ is of the order $1 / n$ (see Proposition 7 in Vives (2011a)).

We study here convergence to the limit continuum market. As $n$ grows large, the equilibria of finite markets, for given information, converge to the limit equilibrium and we characterize the convergence rates for prices and for welfare losses. We find that, for $n$ large, such an equilibrium is close to the equilibrium in the limit economy where traders have no market power and where the price reveals the average parameter $\tilde{\theta}$. Convergence to the equilibrium of the continuum economy occurs as $1 / \sqrt{n}$, the rate at which the average signal $\tilde{S}_{n}$ of the traders (or the average estimate $\mathbb{E}\left[\tilde{\theta}_{n} \mid \tilde{S}_{n}\right]$ ) tends to the average parameter $\tilde{\theta}$ in the continuum economy. Denote by $\mathbb{E}[\mathrm{TS}]$ (resp., $n^{-1} \mathbb{E}\left[\mathrm{TS}_{n}\right]$ ) the per capita expected total surplus in the continuum market (resp., in the $n$-replica markets). The following lemma (with proof in the Appendix) establishes the convergence results.

Lemma 2. Consider the n-replica market. Let $\rho \in[0,1)$. For given $\sigma_{\varepsilon}^{2} \geq 0$, the symmetric DFE of the n-replica market converge to the equilibrium in the continuum economy as $n$ tends to infinity:

(a) $a_{n} \stackrel{n}{\longrightarrow} a, c_{n} \stackrel{n}{\longrightarrow} c$, and $b_{n} \stackrel{n}{\longrightarrow} b$; 
(b) $p_{n}-p \stackrel{n}{\longrightarrow} 0$ in mean square at rate $1 / \sqrt{n}$ with

$$
n \mathbb{E}\left[\left(p_{n}-p\right)^{2}\right] \stackrel{n}{\longrightarrow}\left(\frac{\beta}{\beta+\lambda}\right)^{2} \mathrm{AV},
$$

where $\operatorname{AV}\left(\sigma_{\varepsilon}^{2}\right)=(1-\rho) \sigma_{\theta}^{2}+\sigma_{\varepsilon}^{2}$ if $\rho>0$ and $\operatorname{AV}\left(\sigma_{\varepsilon}^{2}\right)=\sigma_{\theta}^{4}\left(\sigma_{\theta}^{2}+\sigma_{\varepsilon}^{2}\right)^{-1}$ if $\rho=0$

(c) the per capita welfare loss $\mathrm{WL}_{n} \equiv \mathbb{E}[\mathrm{TS}]-n^{-1} \mathbb{E}\left[\mathrm{TS}_{n}\right] \stackrel{n}{\longrightarrow} 0$ at the rate $1 / n$, and the total welfare loss

$$
n \mathrm{WL}_{n} \stackrel{n}{\longrightarrow} \frac{\mathrm{AV}}{2(\beta+\lambda)}+\frac{(1-\rho)^{2} \sigma_{\theta}^{4}}{2 \lambda\left((1-\rho) \sigma_{\theta}^{2}+\sigma_{\varepsilon}^{2}\right)}
$$

The overall convergence result is driven by the rate at which the error terms in the signals vanish, which is slower than the rate of convergence to price-taking behavior. The convergence of the agents' average signal is faster (in terms of asymptotic variance AV; see part (b) of the Lemma) as we approach a common value environment (i.e., as $\rho \rightarrow 1$ ), when there are better signals (low $\sigma_{\varepsilon}^{2}$ for $\rho>0$ ), and/or with less prior uncertainty (low $\left.\sigma_{\theta}^{2}\right)$

In the finite market, the per capita welfare loss (with respect to that in the limit market) is of the order of $1 / n$; see part (c) of Lemma 2. Here again, convergence is faster (in terms of asymptotic variance) when closer to the common value case and slower if there is more prior uncertainty. The effect of noise in the signals is ambiguous if $\rho>0$ since an increase in $\sigma_{\varepsilon}^{2}$ will tend to raise allocative inefficiency while diminish distributive inefficiency. The explanation for those results lies in the expression for total expected welfare loss in the finite market,

$$
n \mathrm{WL}_{n}=n\left((\beta+\lambda) \mathbb{E}\left[\left(\tilde{x}_{n}-\tilde{x}\right)^{2}\right]+\lambda \mathbb{E}\left[\left(u_{i n}-u_{i}\right)^{2}\right]\right) / 2
$$


(where $u_{i n} \equiv x_{i n}-\tilde{x}_{n}$ and $u_{i} \equiv x_{i}-\tilde{x}$ ), which has two components on the right-hand side; the first component reflects allocative inefficiency (is the average quantity at the right level?), and the second reflects distributive inefficiency (is a given average quantity efficiently distributed among market participants?). The first term converges to $\left((1-\rho) \sigma_{\theta}^{2}+\sigma_{\varepsilon}^{2}\right) /(2(\beta+\lambda))$ if $\rho>0$, and the second term converges to $(1-\rho)^{2} \sigma_{\theta}^{4} /\left(2 \lambda\left((1-\rho) \sigma_{\theta}^{2}+\sigma_{\varepsilon}^{2}\right)\right)$ as $n \rightarrow \infty$. Increases in the correlation of parameters $\rho$ or in the precision of the prior $\tau_{\theta} \equiv\left(\sigma_{\theta}^{2}\right)^{-1}$ will decrease both terms; however, the first term increases with $\sigma_{\varepsilon}^{2}$ whereas the second term decreases with $\sigma_{\varepsilon}^{2}$ (since more noise in the signals aligns more individual and average quantities). ${ }^{35}$

The case of an inelastic per capita supply of $q$ is analyzed in the Appendix to illustrate the derivation of equilibrium and its convergence properties as the market grows large. ${ }^{36}$ In this case, a trader bids according to $p=\mathbb{E}\left[\theta_{i} \mid s_{i}, p\right]-\left(d_{n}+\lambda\right) x_{i}$, and competitive bidding obtains when $d=0$. A buyer $\left(x_{i}>0\right)$ underbids, $p<\mathbb{E}\left[\theta_{i} \mid s_{i}, p\right]-\lambda x_{i} ;$ since $d_{n}>0$, a seller $\left(x_{i}<0\right)$ overbids, $p>\mathbb{E}\left[\theta_{i} \mid s_{i}, p\right]-\lambda x_{i}$. We have that $X_{n}\left(s_{i}, p\right) \stackrel{n}{\longrightarrow}(1-\lambda a) q+a\left(s_{i}-p\right)$, the trading strategy in the inelastic supply case in the limit economy.

Reny and Perry (2006), in a finite-market counterpart of their double auction continuum model, prove that, with enough buyers and sellers and with a sufficiently fine grid of prices, generically in the valuation functions of the traders and the fineness of the grid, there is a Bayesian equilibrium in monotonically increasing bid functions that is very close to the unique REE of the continuum economy. The main obstacle in their involved proof is that, with a finite number of traders, in the double auction the strategies of buyers and sellers are not symmetric (despite assuming a symmetry-preserving

\footnotetext{
35 When $\rho=0$, an increase in the noise of the signals reduces both terms.

36

See Vives (2010) for an overview of this model and its properties.
} 
rationing rule). ${ }^{37}$ The incentives of buyers to underbid and of sellers to overbid in order to affect the price disappear as the market grows large and price-taking behavior obtains. In our DFE, the strategy of a trader is symmetric and the trader perceives that her influence on the price is given by $d_{n}>0$. A buyer underbids and a seller overbids, and the incentives to manipulate the market also disappear as $n$ grows and $d_{n} \rightarrow 0$. We can in addition characterize the rate at which this happens (and at which convergence to the limit equilibrium obtains) and distinguish between the effects of dissipation of market power and the averaging of noise terms.

\subsection{Information acquisition in a large market}

Consider the case of covert information acquisition where each trader does not observe the precision purchased by other traders and look therefore at symmetric equilibria of a simultaneous move game where each agent chooses its precision and the demand function $\left(\tau_{\varepsilon_{i}}, X_{i}(\cdot, \cdot)\right)_{i=1, \ldots n}$. At a covert information equilibrium a trader when choosing its precision takes as given both the precisions and the demand functions of the other traders. It is possible to show a parallel result to Proposition 2 in the $n$-replica market.

Let $\psi_{n}\left(\tau_{\varepsilon}\right)$ denote the marginal gross profitability of acquiring precision for a trader at a symmetric solution when others traders purchase $\tau_{\varepsilon}>0$ and the trader follows suit and purchases also $\tau_{\varepsilon}>0$. Interior symmetric equilibria for information precision are characterized then by the solution of $\psi_{n}\left(\tau_{\varepsilon}\right)-H^{\prime}\left(\tau_{\varepsilon}\right)=0$. We have that $\psi_{n}(0) \equiv \lim _{\tau_{\varepsilon} \rightarrow 0} \psi_{n}\left(\tau_{\varepsilon}\right)=\left(2\left(2 d_{n}(0)+\lambda\right) \tau_{\theta}^{2}\right)^{-1}$ where $d_{n}(0) \equiv \lim _{\tau_{\varepsilon} \rightarrow 0} d_{n}\left(\tau_{\varepsilon}\right)$ (and $d_{n}\left(\tau_{\varepsilon}\right)$ is obtained from Lemma 1). Let $\rho<1$, then an interior symmetric equilibrium with $\tau_{\varepsilon}^{*}(n)>0$ exists if $H^{\prime}(0)<\psi_{n}(0)$. For $n$ large, $\psi_{n}\left(\tau_{\varepsilon}\right)$ is strictly decreasing in $\tau_{\varepsilon}$ and

37 The consequence is that the signal of each agent need not be affiliated with the order statistics of the bids of other agents. This failure of "single crossing" implies that standard proofs from auction theory, which rely on relationships between affiliation and order statistics with symmetric strategies, do not apply here. 
therefore a unique interior symmetric equilibrium with $\tau_{\varepsilon}^{*}(n)>0$ exists if and only if $H^{\prime}(0)<\psi_{n}(0)$. Let $\varphi_{n}\left(\tau_{\varepsilon}\right)$ denote the marginal gross profitability of acquiring precision for a trader when the other traders do not acquire information and the trader purchases $\tau_{\varepsilon}$. We have that $\varphi_{n}\left(\tau_{\varepsilon}\right)$ is strictly decreasing in $\tau_{\varepsilon}$ and $\varphi_{n}(0) \equiv \lim _{\tau_{\varepsilon} \rightarrow 0} \varphi_{n}\left(\tau_{\varepsilon}\right)=\left(2\left(2 d_{n}^{f}+\lambda\right) \tau_{\theta}^{2}\right)^{-1}$ (where $d_{n}^{f}$ is the market power distortion with symmetric information). A symmetric equilibrium with $\tau_{\varepsilon}^{*}(n)=0$ exists if and only if $\varphi_{n}(0) \leq H^{\prime}(0)$. Furthermore, $\varphi_{n}(0)>\psi_{n}(0)$ for $\rho>0$ since $d_{n}(0)>d_{n}^{f}$ for $\rho>0$. It follows that for a given $n$ we have that a symmetric equilibrium with $\tau_{\varepsilon}^{*}(n)>0$ exists if $H^{\prime}(0)<\psi_{n}(0)$ and with $\tau_{\varepsilon}^{*}(n)=0$ if $\varphi_{n}(0) \leq H^{\prime}(0)$. In the intermediate region $\psi_{n}(0) \leq H^{\prime}(0)<\varphi_{n}(0)$ there is no symmetric equilibrium for $n$ large. If $\rho=0$, $\psi_{n}(0)=\varphi_{n}(0)$ (since in this case $d_{n}(0)=d_{n}^{f}$ ) and equilibrium always exists. This is the counterpart of Proposition 2 for the finite replica market, stated as Proposition 3 (see the Appendix for a complete proof).

Proposition 3. Consider covert information acquisition in the $n$-replica market. Let $\rho \in[0,1)$. We have that:

(i) If $\varphi_{n}(0) \leq H^{\prime}(0)$, then $\tau_{\varepsilon}^{*}(n)=0$ is the unique symmetric equilibrium.

(ii) If $H^{\prime}(0)<\psi_{n}(0)$, then there is a symmetric equilibrium with $\tau_{\varepsilon}^{*}(n)>0$. It is unique for $n$ large.

(iii) If $\psi_{n}(0) \leq H^{\prime}(0)<\varphi_{n}(0)$, then there is no symmetric equilibrium for $n$ large.

Let us deal now with the large market case. We have that $\psi_{n}\left(\tau_{\varepsilon}\right) \stackrel{n}{\longrightarrow} \psi_{\infty}\left(\tau_{\varepsilon}\right) \equiv(1-\rho)^{2}\left(\tau_{\theta}+(1-\rho) \tau_{\varepsilon}\right)^{-2} / 2 \lambda$, which is the marginal gross profitability of acquiring precision at a symmetric solution in the continuum market. Furthermore, $\varphi_{n}(0) \rightarrow\left(2 \lambda \tau_{\theta}^{2}\right)^{-1}$ from below as $n \rightarrow \infty \quad\left(\varphi_{n}(0) \leq\left(2 \lambda \tau_{\theta}^{2}\right)^{-1}\right.$ since $d_{n}^{f}>0$ 
and $d_{n}^{f} \rightarrow 0$ ). These results provide the basis for the following convergence result (with proof in the Appendix) when information acquisition is endogenous.

Proposition 4. Consider covert information acquisition in the $n$-replica market. Let $\bar{\rho} \equiv 1-\tau_{\theta} \sqrt{2 \lambda H^{\prime}(0)}>0$. Let $\tau_{\varepsilon}^{*}$ denote the equilibrium information precision in the continuum market.

(i) If $\rho \in[0, \bar{\rho})$, then there is a unique symmetric equilibrium $\tau_{\varepsilon}^{*}(n)>0$ for $n$ large and $\tau_{\varepsilon}^{*}(n) \rightarrow \tau_{\varepsilon}^{*}>0$ as $n \rightarrow \infty$. Convergence to the limit equilibrium is "slow": $p_{n}-p \stackrel{n}{\longrightarrow} 0$ in mean square at rate $1 / \sqrt{n}$ with constant of convergence $\mathrm{AV}^{*}=\operatorname{AV}\left(\left(\tau_{\varepsilon}^{*}\right)^{-1}\right)$

(ii) If $\bar{\rho} \leq 0$, then $\tau_{\varepsilon}^{*}=0$ and $\tau_{\varepsilon}^{*}(n)=0$ for any $n$.

Therefore, whenever there is an equilibrium with $\tau_{\varepsilon}^{*}>0$ in the continuum economy it is the limit of equilibria of finite economies. (See Figure 5a.) And whenever there is an equilibrium with $\tau_{\varepsilon}^{*}=0$ in the continuum economy the finite replica markets also call for no information acquisition in equilibrium. ${ }^{38}$

38 However, this need not be the case in the two-stage game where information acquisition is observable at the first stage and $\rho$ is small but positive. The reason is that if in a finite replica market a trader purchases information when other traders do not then this trader affects the market equilibrium and gains some market power which, importantly, does not depend on the amount of information purchased. This will imply that for $\rho>0$ there will be a discontinuity in the marginal benefit to acquire information at 0 for the potentially informed trader. The problem for equilibrium existence may be that the marginal return to information purchase may be negative for any positive precision but then purchasing no precision implies a discrete change to a no information equilibrium where profits are smaller than with some information purchase. See the online Appendix (http://blog.iese.edu/xvives/publications/journal-articles/). 


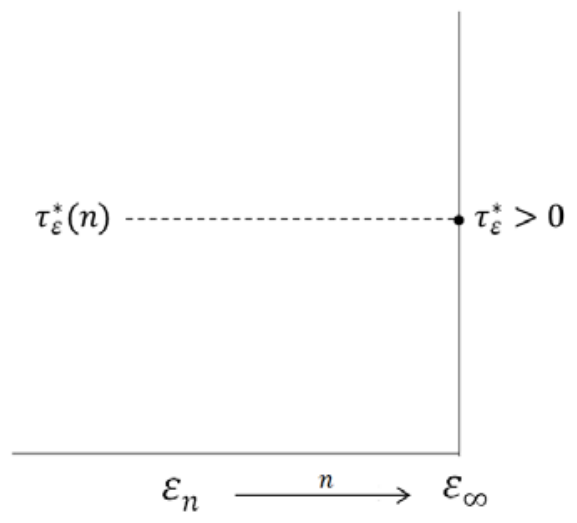

Figure 5.a. Convergence to the equilibrium in the limit market when $H^{\prime}(0)<(1-\rho)^{2}\left(2 \lambda \tau_{\theta}^{2}\right)^{-1}$ or $\rho<\bar{\rho}$.

However, as we shall see, it need not be the case that a sequence of equilibria $\tau_{\varepsilon}^{*}(n)$ in the replica markets always converges to an equilibrium of the limit economy. The reason behind this phenomenon is that the equilibrium correspondence is not continuous in the precision of private information at 0 whenever the correlation parameter $\rho$ is positive. In this case the order in which we take the limits $\tau_{\varepsilon} \rightarrow 0$ and $n \rightarrow \infty$ matters. Indeed, it can be checked that for a given $\tau_{\varepsilon}>0$ we have that $\lim _{n \rightarrow \infty} d_{n}\left(\tau_{\varepsilon}\right)=0$ (and this is the basis for $\psi_{n}\left(\tau_{\varepsilon}\right) \stackrel{n}{\longrightarrow} \psi_{\infty}\left(\tau_{\varepsilon}\right)$ ), and therefore $\lim _{\tau_{\varepsilon} \rightarrow 0} \lim _{n \rightarrow \infty} d_{n}\left(\tau_{\varepsilon}\right)=0$ and there is no market power in the limit. But $\lim _{n \rightarrow \infty} \lim _{\varepsilon} d_{n}\left(\tau_{\varepsilon}\right)=\lim _{n \rightarrow \infty} d_{n}(0)>0$ for $\beta \rho \lambda>0$ and the limit equals 0 for $\beta \rho=0$ (see Claim 1 (i) in the Appendix). In other words, market power $d_{n}\left(\tau_{\varepsilon}\right)$ is not jointly continuous in $\tau_{\varepsilon}$ and $n$ at $\tau_{\varepsilon}=0$ when $\rho>0$.

Equilibrium in the large market may exist while its limit is not an equilibrium of the continuum market.

We show conditions under which we have existence of equilibrium with positive purchase of information $\tau_{\varepsilon}^{*}(n)>0$ with $\lim _{n \rightarrow \infty} \tau_{\varepsilon}^{*}(n)=0$ when $\tau_{\varepsilon}^{*}=0$ is not an equilibrium 
of the continuum economy. ${ }^{39}$ We have that $\psi_{n}(0)=\left(2\left(2 d_{n}(0)+\lambda\right) \tau_{\theta}^{2}\right)^{-1} \leq\left(2 \lambda \tau_{\theta}^{2}\right)^{-1}$. It is possible to show (see Claim 1 (ii) in the Appendix) that there is a unique value of $\beta$, $\hat{\beta}>0$, such that $\lim _{n \rightarrow \infty} \psi_{n}(0)>(1-\rho)^{2}\left(2 \lambda \tau_{\theta}^{2}\right)^{-1}$ if and only if $\beta<\hat{\beta}$, and $\hat{\beta} \rightarrow \infty$ as $\rho \rightarrow 1$. Recall that $\tau_{\varepsilon}^{*}=0$ is an equilibrium of the continuum economy only when $\left(2 \lambda \tau_{\theta}^{2}\right)^{-1} \leq H^{\prime}(0)$. The following proposition provides the result (with proof in the Appendix).

Proposition 5. Let $\beta<\hat{\beta}$. If $\lim _{n \rightarrow \infty} \psi_{n}(0)>H^{\prime}(0) \geq(1-\rho)^{2}\left(2 \lambda \tau_{\theta}^{2}\right)^{-1}$, then for $n$ large there is an equilibrium with $\tau_{\varepsilon}^{*}(n)>0$ with $\lim _{n \rightarrow \infty} \tau_{\varepsilon}^{*}(n)=0$ while there is no equilibrium in the continuum market. The result obtains in particular for $\rho$ close to 1 if $\left(2(2 \beta+\lambda) \tau_{\theta}^{2}\right)^{-1}>H^{\prime}(0)$.

The result for $\rho$ close to 1 obtains since for any $n$, as $\rho \rightarrow 1$ we have that $d_{n}(0) \rightarrow \beta$, and $\psi_{n}(0) \rightarrow\left(2(2 \beta+\lambda) \tau_{\theta}^{2}\right)^{-1}$. Figure $5 \mathrm{~b}$ illustrates this case. Then the region where the Grossman-Stiglitz paradox obtains for $\rho$ close to 1 and $n$ large is reduced from the nonexistence region in the continuum economy $\left(2 \lambda \tau_{\theta}^{2}\right)^{-1}>H^{\prime}(0) \geq(1-\rho)^{2}\left(2 \lambda \tau_{\theta}^{2}\right)^{-1}$.

39 In technical terms this is a failure of upper-hemi continuity of the equilibrium correspondence. 


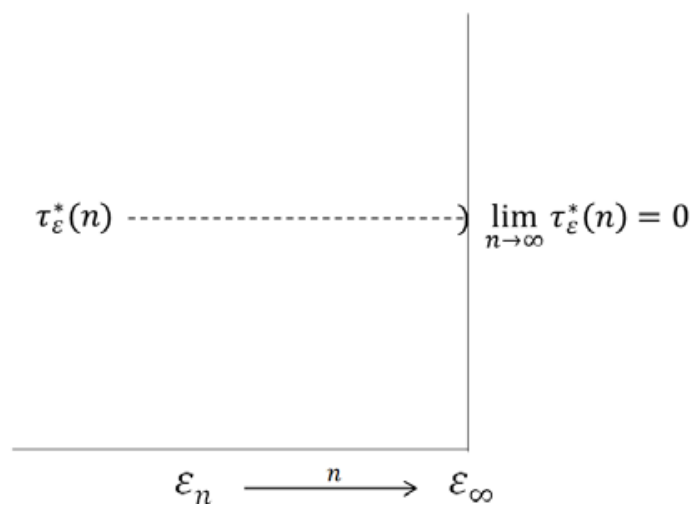

Figure 5.b. Case where the limit of equilibria of finite markets is not an equilibrium in the continuum market. It obtains, for example, when $H^{\prime}(0)<\left(2(2 \beta+\lambda) \tau_{\theta}^{2}\right)^{-1}$ and $\rho$ is close to 1 .

Remark 2: If we allow for non-implementable equilibria in the continuum market then when we have $\tau_{\varepsilon}^{*}(n)>0$ with $\lim _{n \rightarrow \infty} \tau_{\varepsilon}^{*}(n)=0$ it is easily checked that the market equilibrium converges to a fully revealing equilibrium (with $c_{n} \rightarrow-1 / \beta$ ) where $\tau_{\varepsilon}^{*}=0$. This rationalizes the non-implementable fully revealing equilibrium in which no trader purchases information. That is, this fully revealing equilibrium approximates the DFE of a large but finite economy in which traders purchase a little bit of information and the price reveals the average signal $\tilde{s}_{n}$, which is very close to $\tilde{\theta}_{n}$.

\section{Concluding remarks}

A simple large-market REE model which provides conditions to solve the paradoxes associated to fully revealing equilibria has been presented in a context where prices aggregate efficiently information. The key to the resolution of the problems is to allow for both private and common value components in the valuations of the traders, with bundled signals about those components, together with a continuum specification which makes price-taking individually optimal. We have shown that the resolution of the Grossman-Stiglitz paradox is robust to general information structures. Two limitations must be taken into account. Firstly, the resolution of the paradox needs, in general, a bounded common value component for traders' valuation. Second, the continuum 
resolution of the trader's schizophrenia problem comes at the cost of a "slow" convergence rate of equilibrium in finite markets in relation to the rate at which pricetaking behavior obtains.

\section{$\underline{\text { Appendix }}$}

Proof of Proposition 1: Trader $i$ chooses $x_{i}$ to maximize

$$
\mathbb{E}\left[\pi_{i} \mid s_{i}, p\right]=x_{i}\left(\mathbb{E}\left[\theta_{i} \mid s_{i}, p\right]-p\right)-\frac{\lambda}{2} x_{i}^{2}
$$

which yields the FOC $p=\mathbb{E}\left[\theta_{i} \mid s_{i}, p\right]-\lambda x_{i}$. Positing linear strategies $X\left(s_{i}, p\right)=b+a s_{i}-c p$ while using the inverse supply function $p=\alpha+\beta \tilde{x}$ and our convention $\int s_{i} d i=\tilde{\theta}$, we obtain (provided that $1+\beta c \neq 0$ ) an expression for the price $p=(1+\beta c)^{-1}(\alpha+\beta b+\beta a \tilde{\theta})$. The vector $\left(\theta_{i}, s_{i}, \tilde{\theta}\right)$ is normally distributed with $\mathbb{E}\left[\theta_{i}\right]=\mathbb{E}[\tilde{\theta}]=\mathbb{E}\left[s_{i}\right]=\bar{\theta}$ and with variance-covariance matrix

$$
\sigma_{\theta}^{2}\left(\begin{array}{ccc}
1 & 1 & \rho \\
1 & \xi^{-1} & \rho \\
\rho & \rho & \rho
\end{array}\right),
$$

where $\quad \xi=\tau_{\varepsilon} /\left(\tau_{\theta}+\tau_{\varepsilon}\right)$. It follows that $\mathbb{E}\left[\theta_{i} \mid s_{i}, \tilde{\theta}\right]=\varsigma s_{i}+(1-\varsigma) \tilde{\theta} \quad$ for $\varsigma=\left(1+\sigma_{\varepsilon}^{2} /(1-\rho) \sigma_{\theta}^{2}\right)^{-1}$. Given joint normality of the stochastic variables $\left(\theta_{i}, s_{i}, \tilde{\theta}\right)$, we obtain

$$
\left(\begin{array}{c}
\theta_{i} \\
s_{i} \\
p
\end{array}\right) \sim N\left(\left(\begin{array}{c}
\bar{\theta} \\
\bar{\theta} \\
C+D \bar{\theta}
\end{array}\right),\left(\begin{array}{ccc}
\sigma_{\theta}^{2} & \sigma_{\theta}^{2} & D \rho \sigma_{\theta}^{2} \\
\sigma_{\theta}^{2} & \sigma_{\theta}^{2}+\sigma_{\varepsilon}^{2} & D \rho \sigma_{\theta}^{2} \\
D \rho \sigma_{\theta}^{2} & D \rho \sigma_{\theta}^{2} & D^{2} \rho \sigma_{\theta}^{2}
\end{array}\right)\right) .
$$

Here $C=(1+\beta c)^{-1}(\alpha+\beta b)$ and $D=(1+\beta c)^{-1}(\beta a)$. If we use the projection theorem for normal random variables and assume that $\beta a \neq 0$, then 


$$
\mathbb{E}\left[\theta_{i} \mid s_{i}, p\right]=-\frac{C \sigma_{\varepsilon}^{2}}{D\left((1-\rho) \sigma_{\theta}^{2}+\sigma_{\varepsilon}^{2}\right)}+\frac{(1-\rho) \sigma_{\theta}^{2}}{(1-\rho) \sigma_{\theta}^{2}+\sigma_{\varepsilon}^{2}} s_{i}+\frac{\sigma_{\varepsilon}^{2}}{D\left((1-\rho) \sigma_{\theta}^{2}+\sigma_{\varepsilon}^{2}\right)} p .
$$

Using the first-order condition, we obtain

$$
\begin{aligned}
\frac{\sigma_{\varepsilon}^{2}}{\left(\sigma_{\varepsilon}^{2}+(1-\rho) \sigma_{\theta}^{2}\right)} \frac{\alpha+\beta b}{\beta a} & -\frac{(1-\rho) \sigma_{\theta}^{2}}{\left(\sigma_{\varepsilon}^{2}+(1-\rho) \sigma_{\theta}^{2}\right)} s_{i}+\left(1-\frac{\sigma_{\varepsilon}^{2}}{\left(\sigma_{\varepsilon}^{2}+(1-\rho) \sigma_{\theta}^{2}\right)} \frac{1+\beta c}{\beta a}\right) p \\
& =-\lambda\left(b+a s_{i}-c p\right) ;
\end{aligned}
$$

then, using the method of undetermined coefficients, we obtain the following system of equations:

$$
\left.\begin{array}{c}
\frac{(1-\rho) \sigma_{\theta}^{2}}{\lambda\left(\sigma_{\varepsilon}^{2}+(1-\rho) \sigma_{\theta}^{2}\right)}=a \\
-\frac{\sigma_{\varepsilon}^{2}}{\left(\sigma_{\varepsilon}^{2}+(1-\rho) \sigma_{\theta}^{2}\right)} \frac{\alpha+\beta b}{\beta a}=\lambda b \\
1-\frac{\sigma_{\varepsilon}^{2}}{\left(\sigma_{\varepsilon}^{2}+(1-\rho) \sigma_{\theta}^{2}\right)} \frac{(1+\beta c)}{\beta a}=\lambda c
\end{array}\right\}
$$

The solution to this system gives the result because for $\rho \in(0,1), \sigma_{\theta}^{2}>0$, and $\sigma_{\varepsilon}^{2}<\infty$ we have that $1+\beta c=a(\beta+\lambda)>0$, and

$$
\begin{aligned}
& a=\frac{1}{\lambda\left(1+\left(\sigma_{\varepsilon}^{2} /\left((1-\rho) \sigma_{\theta}^{2}\right)\right)\right)}=\frac{1}{\lambda(1+M)}, \\
& b=-\frac{\alpha}{\beta} \frac{\sigma_{\varepsilon}^{2}}{\left(\sigma_{\varepsilon}^{2}+(1-\rho) \sigma_{\theta}^{2}\right)}=-\frac{\alpha}{\beta}(1-\lambda a), \\
& c=\frac{\lambda^{-1}(1-\rho) \sigma_{\theta}^{2}-\beta^{-1} \sigma_{\varepsilon}^{2}}{\left(\sigma_{\varepsilon}^{2}+(1-\rho) \sigma_{\theta}^{2}\right)}=\frac{1}{\beta}(a(\beta+\lambda)-1) .
\end{aligned}
$$

It is immediate that $a>0$, that $-\beta^{-1}<c \leq a \leq \lambda^{-1}$ for $\beta>0$, and that $c$ decreases in $M$ and $\lambda$ but increases in $\beta$. Finally, we can use $p=(1+\beta c)^{-1}(\alpha+\beta b+\beta a \tilde{\theta})$ together with the expressions for the equilibrium coefficients to show that $p=(\lambda \alpha+\beta \tilde{\theta}) /(\lambda+\beta)$ and $\tilde{x}=(\tilde{\theta}-\alpha) /(\beta+\lambda)$. 
Proposition $2 *$ Let $\rho \in[0,1]$ and $\bar{\rho} \equiv 1-\tau_{\theta} \sqrt{2 \lambda H^{\prime}(0)}$ and consider the information structure with signal $s_{i}=\eta_{i}+\gamma \tilde{\theta}+\omega+\varepsilon_{i}$. We have that:

(i) If $(1+\rho(\gamma-1))^{2}\left(2 \lambda \tau_{\theta}^{2}\right)^{-1} \leq H^{\prime}(0)$, then $\tau_{\varepsilon}^{*}=0$.

(ii) If $H^{\prime}(0)<(1-\rho)^{2}\left(2 \lambda \tau_{\theta}^{2}\right)^{-1}$, or equivalently $\bar{\rho}>\rho \geq 0$, then $\tau_{\varepsilon}^{*}>0$ is an equilibrium and $\tau_{\varepsilon}^{*}$ is decreasing in $\lambda, \tau_{\theta}$, and $\rho$.

(iii) If $(1+\rho(\gamma-1))^{2}\left(2 \lambda \tau_{\theta}^{2}\right)^{-1}>H^{\prime}(0) \geq(1-\rho)^{2}\left(2 \lambda \tau_{\theta}^{2}\right)^{-1}$ there is no equilibrium.

This region exists if and only if $\rho \gamma>0$, and it grows with $\gamma$, and with $\rho$ if $\gamma \geq 1$.

\begin{tabular}{|c|c|c|c|c|c|}
\hline \multirow[b]{2}{*}{0} & \multicolumn{2}{|l|}{$\tau_{\varepsilon}^{*}>0$} & $\begin{array}{c}\text { No equilibrium } \\
\text { (G-S paradox) }\end{array}$ & \multicolumn{2}{|c|}{$\tau_{\varepsilon}^{*}=0$} \\
\hline & $(\bar{\rho}>\rho)$ & $(1-\rho)^{2}$ & $(\rho \geq \bar{\rho})$ & $(1+\rho(\gamma-1))^{2}$ & $\overrightarrow{H^{\prime}(0)}$ \\
\hline & & $\overline{2 \lambda \tau_{\theta}^{2}}$ & & $2 \lambda \tau_{\theta}^{2}$ & \\
\hline
\end{tabular}

Figure A. The range of the Grossman-Stiglitz paradox when $\gamma>0$.

Proof: Suppose that, at the first stage, all traders but $i$ have chosen a precision $\tau_{\varepsilon}>0$. Then the market equilibrium (which is unaffected by the actions of a single trader) reveals $\tilde{s}=\gamma \tilde{\theta}+\omega$. Therefore $\mathbb{E}\left[\theta_{i} \mid s_{i}, p\right]=\mathbb{E}\left[\theta_{i} \mid \hat{s}_{i}, \tilde{s}\right]$, where $\hat{s}_{i}=\eta_{i}+\varepsilon_{i}$. Trader $i$ receives a signal with precision $\tau_{\varepsilon_{i}}$ and chooses $x_{i}$ to maximize

$$
\left(\mathbb{E}\left[\theta_{i} \mid \hat{s}_{i}, \tilde{s}\right]-p\right) x_{i}-\frac{\lambda}{2} x_{i}^{2}
$$

which yields the first order condition $\mathbb{E}\left[\theta_{i} \mid \hat{s}_{i}, \tilde{s}\right]-p=\lambda x_{i}$. Expected profits are given by $\mathbb{E}\left[\pi_{i}\right]=(\lambda / 2) \mathbb{E}\left[x_{i}^{2}\right]$. Note that $\mathbb{E}\left[\pi_{i}\right]$ does not depend on $\tau_{\varepsilon}$ because the equilibrium reveals $\tilde{s}$. We notice that $\mathbb{E}\left[\theta_{i} \mid \hat{s}_{i}, \tilde{s}\right]=\mathbb{E}\left[\theta_{i} \mid \hat{s}_{i}, \tilde{s}^{\prime}\right]$, where $\tilde{s}^{\prime}=\gamma^{-1} \tilde{s}=\tilde{\theta}+\omega \gamma^{-1}$. The vector $\left(\theta_{i}, \hat{s}, \tilde{s}^{\prime}\right)$ has mean $(\bar{\theta}, 0, \bar{\theta})$ and variance-covariance matrix 


$$
\left(\begin{array}{ccc}
\tau_{\theta}^{2} & (1-\rho) \sigma_{\theta}^{2} & \rho \sigma_{\theta}^{2} \\
(1-\rho) \sigma_{\theta}^{2} & (1-\rho) \sigma_{\theta}^{2}+\sigma_{\varepsilon_{i}}^{2} & 0 \\
\rho \sigma_{\theta}^{2} & 0 & \sigma_{\theta}^{2} \rho+\gamma^{-2} \sigma_{\omega}^{2}
\end{array}\right)
$$

Applying the projection theorem, we obtain that $\mathbb{E}\left[\theta_{i} \mid \hat{s}_{i}, \tilde{s}^{\prime}\right]=\bar{\theta}(1-\kappa)+\varsigma_{i} \hat{s}_{i}+\kappa \tilde{s}^{\prime}$ where $\varsigma_{i}=\left(\tau_{\varepsilon_{i}}(1-\rho)\right) /\left((1-\rho) \tau_{\varepsilon_{i}}+\tau_{\theta}\right)$ and $\kappa=\rho \tau_{\omega} /\left(\rho \tau_{\omega}+\gamma^{-2} \tau_{\theta}\right)$. Note that when $\tau_{\omega} \rightarrow \infty, \kappa \rightarrow 1$ and the conditional expectation becomes $\mathbb{E}\left[\theta_{i} \mid \hat{s}_{i}, \tilde{s}^{\prime}\right]=\varsigma_{i}\left(s_{i}-\tilde{\theta}\right)+\tilde{\theta}$ since $\hat{s}_{i}=s_{i}-\tilde{\theta}$ and $\tilde{s}^{\prime}=\tilde{\theta}$. (in the base model we have also that $\gamma=1$.)

Using the first order condition and market clearing condition, we find the expression for the price. Notice that both conditions imply that $(p-\alpha) \beta^{-1}=\left(\int \mathbb{E}\left[\theta_{j} \mid \hat{s}_{j}, \tilde{s}^{\prime}\right] d j-p\right) \lambda^{-1}$ where $\quad \int \mathbb{E}\left[\theta_{j} \mid \hat{s}_{j}, \tilde{s}^{\prime}\right] d j=\bar{\theta}(1-\kappa)+\varsigma \int \hat{s}_{j} d j+\kappa \tilde{s}^{\prime}=\bar{\theta}(1-\kappa)+\kappa \tilde{s}^{\prime} \quad, \quad$ where $\varsigma=\left(\tau_{\varepsilon}(1-\rho)\right) /\left((1-\rho) \tau_{\varepsilon}+\tau_{\theta}\right)$, since $\int \hat{s}_{j} d j=0$. Hence, the expression from the price can be written as:

$$
p=\frac{\alpha \lambda+\bar{\theta}(1-\kappa) \beta+\beta \kappa \gamma^{-1} \tilde{s}}{(\lambda+\beta)}
$$

Substituting for the expression of the price, we find that

$$
x_{i}=\frac{1}{\lambda}\left(\bar{\theta}(1-\kappa)+\varsigma_{i} \hat{s}_{i}+\frac{\lambda \kappa \gamma^{-1} \tilde{s}}{\lambda+\beta}-\frac{\bar{\theta}(1-\kappa) \beta+\alpha \lambda}{\lambda+\beta}\right) .
$$

From the laws of expectations, we note that $\mathbb{E}\left[x_{i}^{2}\right]=\operatorname{var}\left[x_{i}\right]+\left(\mathbb{E}\left[x_{i}\right]\right)^{2}$, where $\mathbb{E}\left[x_{i}\right]=(\bar{\theta}-\alpha) /(\lambda+\beta)$ and, since $\operatorname{cov}\left[\hat{s}_{i}, \tilde{s}\right]=0$, $\operatorname{var}\left[x_{i}\right]=\frac{1}{\lambda^{2}}\left(\varsigma_{i}^{2} \operatorname{var}\left[\hat{s}_{i}\right]+\frac{\lambda^{2} \kappa^{2}}{(\lambda+\beta)^{2}} \operatorname{var}[\tilde{s}]\right)=\frac{1}{\lambda^{2}}\left(\frac{\tau_{\varepsilon_{i}}(1-\rho)^{2}}{\left((1-\rho) \tau_{\varepsilon_{i}}+\tau_{\theta}\right) \tau_{\theta}}+\frac{\lambda^{2}}{(\lambda+\beta)^{2}} \frac{\rho^{2} \tau_{u}}{\left(\rho \tau_{\omega}+\tau_{\theta} \gamma^{-2}\right) \tau_{\theta}}\right)$

We can use this fact to obtain

$$
\mathbb{E}\left[\pi_{i}\right]=\frac{\lambda}{2} \frac{(\bar{\theta}-\alpha)^{2}}{(\lambda+\beta)^{2}}+\frac{1}{2 \lambda \tau_{\theta}}\left(\frac{\tau_{\varepsilon_{i}}(1-\rho)^{2}}{(1-\rho) \tau_{\varepsilon_{i}}+\tau_{\theta}}+\frac{\lambda^{2}}{(\lambda+\beta)^{2}} \frac{\rho^{2} \tau_{\omega}}{\rho \tau_{\omega}+\tau_{\theta} \gamma^{-2}}\right) .
$$


It follows that the marginal benefit of increasing the precision of information is

$$
\frac{\partial \mathbb{E}\left[\pi_{i}\right]}{\partial \tau_{\varepsilon_{i}}}=\frac{1}{2 \lambda} \frac{(1-\rho)^{2}}{\left((1-\rho) \tau_{\varepsilon_{i}}+\tau_{\theta}\right)^{2}},
$$

which is independent of $\gamma$ or $\tau_{\omega}$ because $\operatorname{cov}\left[\hat{s}_{i}, \tilde{s}\right]=0$. Observe that this marginal benefit is decreasing in $\tau_{\varepsilon_{i}}$ provided that $\rho<1$ (and thus $\mathbb{E}\left[\pi_{i}\right]$ is strictly concave in $\tau_{\varepsilon_{i}}$ ). Let

$$
\phi\left(\tau_{\varepsilon}\right)=\left.\frac{\partial \mathbb{E}\left[\pi_{i}\right]}{\partial \tau_{\varepsilon_{i}}}\right|_{\tau_{\varepsilon_{i}}=\tau_{\varepsilon}}-H^{\prime}\left(\tau_{\varepsilon}\right)
$$

Then $\phi(\infty)<0$ and $\phi^{\prime}<0$. We have that $\phi(0)>0$ if and only if $\rho<\bar{\rho}=1-\tau_{\theta} \sqrt{2 \lambda H^{\prime}(0)}$, in which there is a unique interior solution $\tau_{\varepsilon}^{*}$ to the equation $\phi\left(\tau_{\varepsilon}\right)=0$. Note that $\tau_{\varepsilon}^{*}$ is decreasing in $\lambda, \tau_{\theta}$, and $\rho$ because $\phi$ is.

For $\rho \geq \bar{\rho}$ we have that $\tau_{\varepsilon}=0$ at a candidate equilibrium. When no trader other than $i$ purchases information, then the price $p=(\lambda \alpha+\beta \bar{\theta}) /(\lambda+\beta)$ conveys no information and we can check that

$$
\mathbb{E}\left[\theta_{i} \mid s_{i}\right]=\bar{\theta}+\frac{(1+\rho(\gamma-1)) \tau_{\omega} \tau_{\varepsilon_{i}}}{\left(1+\rho\left(\gamma^{2}-1\right)\right) \tau_{\omega} \tau_{\varepsilon_{i}}+\tau_{\omega} \tau_{\theta}+\tau_{\theta} \tau_{\varepsilon_{i}}}\left(s_{i}-\bar{\theta}\right),
$$

where $s_{i}=\eta_{i}+\gamma \tilde{\theta}+\omega+\varepsilon_{i}$. Then, we have

$$
x_{i}=\frac{1}{\lambda}\left(\bar{\theta}+\frac{(1+\rho(\gamma-1)) \tau_{\omega} \tau_{\varepsilon_{i}}}{\left(1+\rho\left(\gamma^{2}-1\right)\right) \tau_{\omega} \tau_{\varepsilon_{i}}+\tau_{\omega} \tau_{\theta}+\tau_{\theta} \tau_{\varepsilon_{i}}}\left(s_{i}-\bar{\theta}\right)-\frac{\lambda \alpha+\beta \bar{\theta}}{\lambda+\beta}\right) .
$$

As before $\mathbb{E}\left[x_{i}^{2}\right]=\operatorname{var}\left[x_{i}\right]+\left(\mathbb{E}\left[x_{i}\right]\right)^{2}$ and $\mathbb{E}\left[x_{i}\right]=(\bar{\theta}-\alpha) /(\lambda+\beta)$. We obtain that 


$$
\operatorname{var}\left[x_{i}\right]=\frac{1}{\lambda^{2} \tau_{\theta}}\left(\frac{(1+\rho(\gamma-1))^{2} \tau_{\omega} \tau_{\varepsilon_{i}}}{\left(1+\rho\left(\gamma^{2}-1\right)\right) \tau_{\omega} \tau_{\varepsilon_{i}}+\tau_{\omega} \tau_{\theta}+\tau_{\theta} \tau_{\varepsilon_{i}}}\right) .
$$

Then expected profits are

$$
\mathbb{E}\left[\pi_{i}\right]=\frac{\lambda}{2}\left(\frac{\bar{\theta}-\alpha}{\lambda+\beta}\right)^{2}+\frac{1}{2 \lambda \tau_{\theta}} \frac{(1+\rho(\gamma-1))^{2} \tau_{\omega} \tau_{\varepsilon_{i}}}{\left(1+\rho\left(\gamma^{2}-1\right)\right) \tau_{\omega} \tau_{\varepsilon_{i}}+\tau_{\omega} \tau_{\theta}+\tau_{\theta} \tau_{\varepsilon_{i}}}
$$

and

$$
\frac{\partial \mathbb{E}\left[\pi_{i}\right]}{\partial \tau_{\varepsilon_{i}}}=\frac{(1+\rho(\gamma-1))^{2}}{2 \lambda} \frac{\tau_{\omega}^{2}}{\left((1+\rho(\gamma-1)) \tau_{\omega} \tau_{\varepsilon_{i}}+\tau_{\omega} \tau_{\theta}+\tau_{\theta} \tau_{\varepsilon_{i}}\right)^{2}} .
$$

It follows that $\tau_{\varepsilon}=0$ is an equilibrium only if $(1+\rho(\gamma-1))^{2}\left(2 \lambda \tau_{\theta}{ }^{2}\right)^{-1} \leq H^{\prime}(0)$. Otherwise (i.e. if $\left.(1+\rho(\gamma-1))^{2}\left(2 \lambda \tau_{\theta}^{2}\right)^{-1}>H^{\prime}(0) \geq(1-\rho)^{2}\left(2 \lambda \tau_{\theta}^{2}\right)^{-1}\right)$ there is no (symmetric) equilibrium. This region exists if and only if $\gamma>0$ and $\rho>0$. The length of this region is given by $l=\left((1-\rho+\rho \gamma)^{2}-(1-\rho)^{2}\right) /\left(2 \lambda \tau_{\theta}^{2}\right)$ it and grows with $\gamma$ since $\partial l / \partial \gamma=\left(\rho^{2} \gamma+(1-\rho)\right) /\left(\lambda \tau_{\theta}^{2}\right)>0 \quad$ and $\quad$ with $\quad \rho, \quad$ whenever $\quad \gamma \geq 1$ since $\partial l / \partial \rho=\gamma((1-\rho)+\rho(\gamma-1)) /\left(\lambda \tau_{\theta}^{2}\right)$.

When $\gamma<0$, then $(1-\rho)^{2}>(1+\rho(\gamma-1))^{2}$. There is an interior solution, $\tau_{\varepsilon}^{*}>0$, when $\quad(1-\rho)^{2}\left(2 \lambda \tau_{\theta}^{2}\right)^{-1}>H^{\prime}(0)$ and $\quad \tau_{\varepsilon}=0 \quad$ is an equilibrium only if $H^{\prime}(0) \geq(1+\rho(\gamma-1))^{2}\left(2 \lambda \tau_{\theta}^{2}\right)^{-1} \quad$. Hence for the region $(1-\rho)^{2}\left(2 \lambda \tau_{\theta}^{2}\right)^{-1}>H^{\prime}(0) \geq(1+\rho(\gamma-1))^{2}\left(2 \lambda \tau_{\theta}{ }^{2}\right)^{-1}$ there are multiple equilibria, one with and another without information acquisition.

Proposition 2 follows letting $\kappa=1$ (it holds also for any value of $\tau_{\omega}$ ). 
Lemma A. If $H^{\prime}(0)<(1-\rho)^{2}\left(2 \lambda \tau_{\theta}^{2}\right)^{-1}$ the marginal social and private benefits to purchase information are the same and the market acquires the right amount of information $\tau_{\varepsilon}^{*}>0$. When $H^{\prime}(0) \geq(1-\rho)^{2}\left(2 \lambda \tau_{\theta}^{2}\right)^{-1}$ and $\rho>0$ there is no welfareoptimal level of information purchase.

Proof: Gross expected total surplus is given by

$$
\mathbb{E}[\mathrm{TS}]=\mathbb{E}\left[\int_{0}^{1}\left(\theta_{i} x_{i}-\frac{\lambda}{2} x_{i}^{2}\right) d i-\left(\alpha+\beta \frac{\tilde{x}}{2}\right) \tilde{x}\right],
$$

which at the market allocation we have that $x_{i}$ depends on $\left(s_{i}, \tilde{\theta}\right)$ and $\tilde{x}$ on $\tilde{\theta} . \mathbb{E}[T S]$ only depends on $\tau_{\varepsilon}$ from $\mathbb{E}\left[\theta_{i} x_{i}\right]-(\lambda / 2) \mathbb{E}\left[x_{i}^{2}\right]$. Some computations lead to

$$
\mathbb{E}\left[\theta_{i} x_{i}\right]=\bar{\theta}\left(\frac{\bar{\theta}-\alpha}{\lambda+\beta}\right)+\sigma_{\theta}^{2} \frac{\beta(1-\rho)+\lambda(M \rho+1)}{\lambda(\beta+\lambda)(M+1)}
$$

and using the expression for $\mathbb{E}\left[x_{i}^{2}\right]$ in the proof of Proposition $2^{*}$ letting $\gamma=1$ we obtain that

$$
\frac{\partial \mathbb{E}[\mathrm{TS}]}{\partial \tau_{\varepsilon}}=\frac{(1-\rho)^{2}}{2 \lambda\left(\tau_{\theta}+(1-\rho) \tau_{\varepsilon}\right)^{2}},
$$

which is exactly the same as the marginal profit obtained in the market solution. We have similarly that at the market equilibrium $\mathbb{E}[\mathrm{TS}]$ is strictly concave in $\tau_{\varepsilon}$ and therefore the interior solutions $\phi\left(\tau_{\varepsilon}\right)=0$, when $H^{\prime}(0)<(1-\rho)^{2}\left(2 \lambda \tau_{\theta}^{2}\right)^{-1}$, will coincide with the market.

However, when $H^{\prime}(0) \geq(1-\rho)^{2}\left(2 \lambda \tau_{\theta}^{2}\right)^{-1}$ and $\rho>0$ there is no welfare-optimal level of information purchase because the maximization of $\mathbb{E}[\mathrm{TS}]-H\left(\tau_{\varepsilon}\right)$ becomes an open problem. When $H^{\prime}(0) \geq(1-\rho)^{2}\left(2 \lambda \tau_{\theta}^{2}\right)^{-1}, \mathbb{E}[\mathrm{TS}]$ is decreasing in $\tau_{\varepsilon}$ for $\tau_{\varepsilon}>0$ but setting $\tau_{\varepsilon}=0$ delivers strictly less surplus than a small positive $\tau_{\varepsilon}$ since for $\rho>0$, 
$\lim _{\tau_{\varepsilon} \rightarrow 0^{+}} \mathbb{E}[T S]=\frac{1}{2} \frac{(\bar{\theta}-\alpha)^{2}+\rho \sigma_{\theta}^{2}}{\beta+\lambda}>\left.\mathbb{E}[T S]\right|_{\tau_{\varepsilon}=0}=\frac{1}{2} \frac{(\bar{\theta}-\alpha)^{2}}{\beta+\lambda}$

Measures of speed of convergence. We say that the sequence (of real numbers) $b_{n}$ is of the order $n^{v}$ ( $v$ a real number) whenever $n^{-v} b_{n} \underset{n}{\longrightarrow} k$ for some nonzero constant $k .{ }^{40}$ The constant of convergence $k$ is a refined measure of the speed of convergence. We say that the sequence of random variables $\left\{y_{n}\right\}$ converges in mean square to zero at the rate $1 / \sqrt{n^{r}}$ (or that $y_{n}$ is of the order $1 / \sqrt{n^{r}}$ ) if $\mathbb{E}\left[\left(y_{n}\right)^{2}\right]$ converges to zero at the rate $1 / n^{r}$ (i.e., if $\mathbb{E}\left[\left(y_{n}\right)^{2}\right]$ is of the order $1 / n^{r}$ ). Given that $\mathbb{E}\left[\left(y_{n}\right)^{2}\right]=\left(\mathbb{E}\left[y_{n}\right]\right)^{2}+\operatorname{var}\left[y_{n}\right]$, a sequence $\left\{y_{n}\right\}$ such that $\mathbb{E}\left[y_{n}\right]=0$ and $\operatorname{var}\left[y_{n}\right]$ is of order $1 / n$ and converges to zero at the rate $1 / \sqrt{n}$. A more refined measure of the speed of convergence for a given convergence rate is provided by the asymptotic variance. Suppose that $\mathbb{E}\left[\left(y_{n}\right)^{2}\right] \stackrel{n}{\longrightarrow} 0$ at the rate $1 / n^{r}$ and $\mathbb{E}\left[y_{n}\right]=0$. Then the asymptotic variance of convergence is given by the constant $\lim _{n \rightarrow \infty} n^{r} \mathbb{E}\left[\left(y_{n}\right)^{2}\right]$. A higher asymptotic variance means that the speed of convergence is slower.

Proof of Lemma 2: (a) From the proof of Proposition 7 in Vives (2011b) we have that $c_{n} \stackrel{n}{\longrightarrow} C \equiv\left(\lambda^{-1}-\beta^{-1} M\right) /(M+1)$, where $M=\sigma_{\varepsilon}^{2}(1-\rho)^{-1} \sigma_{\theta}^{-2}$ if $\rho>0$ and $c_{n} \stackrel{n}{\longrightarrow} \lambda^{-1}$ if $\rho=0$.

Furthermore, $\quad a_{n}=(1-\rho) \sigma_{\theta}^{2}\left(\sigma_{\varepsilon}^{2}+(1-\rho) \sigma_{\theta}^{2}\right)^{-1}\left(d_{n}+\lambda\right)^{-1} \stackrel{n}{\longrightarrow} a \quad$ because $d_{n}=\left(\beta^{-1} n+(n-1) c_{n}\right)^{-1} \stackrel{n}{\longrightarrow} 0$. Note that $n d_{n} \stackrel{n}{\longrightarrow}\left(\beta^{-1}+c\right)^{-1}$, which is equal to 40 This definition is stronger than necessary but it will suffice for our purposes. 
$\left(\beta^{-1}+\lambda^{-1}\right)^{-1}(1+M)$ if $\rho>0$ or to $\left(\beta^{-1}+\lambda^{-1}\right)^{-1}$ if $\rho=0$. Convergence for $b_{n}$ follows similarly.

(b) From Proposition 1 in Vives (2011a) we have that $\tilde{x}_{n}=\left(\mathbb{E}\left[\tilde{\theta}_{n} \mid \tilde{s}_{n}\right]-\alpha\right) /\left(\beta+\lambda+d_{n}\right)$, where $\quad \tilde{s}_{n}=n^{-1}\left(\sum_{i} s_{i}\right)=\tilde{\theta}_{n}+n^{-1}\left(\sum_{i} \varepsilon_{i}\right) \quad, \quad \mathbb{E}\left[\tilde{\theta}_{n} \mid \tilde{s}_{n}\right]=\zeta_{n} \tilde{s}_{n}+\left(1-\zeta_{n}\right) \bar{\theta} \quad, \quad$ and $\zeta_{n} \equiv \operatorname{var}\left[\tilde{\theta}_{n}\right] /\left(\operatorname{var}\left[\tilde{\theta}_{n}\right]+\sigma_{\varepsilon}^{2} n^{-1}\right)$. It follows that

$$
\operatorname{var}\left[\mathbb{E}\left[\tilde{\theta}_{n} \mid \tilde{S}_{n}\right]\right]=\zeta_{n} \operatorname{var}\left[\tilde{\theta}_{n}\right]=\frac{\left((1+(n-1) \rho) \sigma_{\theta}^{2}\right)^{2}}{\left((1+(n-1) \rho) \sigma_{\theta}^{2}+\sigma_{\varepsilon}^{2}\right) n} .
$$

Observe that $d_{n} \stackrel{n}{\longrightarrow} 0$ and $\mathbb{E}\left[\tilde{\theta}_{n} \mid \tilde{s}_{n}\right] \stackrel{n}{\longrightarrow} \tilde{\theta}$ in mean square (since $\tilde{\theta}_{n} \stackrel{n}{\longrightarrow} \tilde{\theta}$ and $\left(\sum_{i} \varepsilon_{i}\right) / n \stackrel{n}{\longrightarrow} 0$ in mean square, both at rate $\left.1 / \sqrt{n}\right)$. It is immediate that

$$
\mathbb{E}\left[\left(\tilde{\theta}-\mathbb{E}\left[\tilde{\theta}_{n} \mid \tilde{S}_{n}\right]\right)^{2}\right]=\frac{\sigma_{\theta}^{2}(1-\rho)(\rho(n-1)+1)+n \rho \sigma_{\varepsilon}^{2}}{n\left(\sigma_{\theta}^{2}+\sigma_{\varepsilon}^{2}+(n-1) \rho \sigma_{\theta}^{2}\right)} \sigma_{\theta}^{2}
$$

and

$n \mathbb{E}\left[\left(\tilde{\theta}-\mathbb{E}\left[\tilde{\theta}_{n} \mid \tilde{s}_{n}\right]\right)^{2}\right] \stackrel{n}{\longrightarrow} \mathrm{AV} \quad, \quad$ where $\quad \mathrm{AV}=(1-\rho) \sigma_{\theta}^{2}+\sigma_{\varepsilon}^{2} \quad$ if $\quad \rho>0 \quad$ and $\mathrm{AV}=\sigma_{\theta}^{4}\left(\sigma_{\theta}^{2}+\sigma_{\varepsilon}^{2}\right)^{-1}$ if $\rho=0$. We have that $\tilde{x}=(\tilde{\theta}-\alpha) /(\beta+\lambda)$, that

$$
\mathbb{E}\left[\left(\tilde{x}-\tilde{x}_{n}\right)^{2}\right]=\mathbb{E}\left[\left(\frac{(\beta+\lambda)\left(\tilde{\theta}-\mathbb{E}\left[\tilde{\theta}_{n} \mid \tilde{s}_{n}\right]\right)+d_{n}(\tilde{\theta}-\alpha)}{(\beta+\lambda)\left(\beta+\lambda+d_{n}\right)}\right)^{2}\right],
$$

and that both $d_{n}$ and $\mathbb{E}\left[\left(\tilde{\theta}-\mathbb{E}\left[\tilde{\theta}_{n} \mid \tilde{s}_{n}\right]\right)^{2}\right]$ are of order $1 / n$; hence we obtain $n \mathbb{E}\left[\left(\tilde{x}-\tilde{x}_{n}\right)^{2}\right] \stackrel{n}{\longrightarrow} \mathrm{AV} /(\beta+\lambda)^{2}$. Therefore, $\tilde{x}-\tilde{x}_{n} \stackrel{n}{\longrightarrow} 0$ in mean square. The results follow since $p_{n}-p=\beta\left(\tilde{x}_{n}-\tilde{x}\right)$.

(c) Total surplus (per capita) in the continuum and in the n-replica markets are given, respectively, by 


$$
\mathrm{TS}=\int_{0}^{1}\left(\theta_{i} x_{i}-\frac{\lambda}{2} x_{i}^{2}\right) d i-\left(\alpha+\beta \frac{\tilde{x}}{2}\right) \tilde{x} \quad \text { and } \quad n^{-1} \mathrm{TS}_{n}=n^{-1} \sum_{i=1}^{n}\left(\theta_{i} x_{i}-\frac{\lambda}{2} x_{i}^{2}\right)-\left(\alpha+\beta \frac{\tilde{x}_{n}}{2}\right) \tilde{x}_{n}
$$

We can then write the expected welfare loss as

$$
\mathrm{WL}_{n} \equiv \mathbb{E}[\mathrm{TS}]-n^{-1} \mathbb{E}\left[\mathrm{TS}_{n}\right]=\left((\beta+\lambda) \mathbb{E}\left[\left(\tilde{x}_{n}-\tilde{x}\right)^{2}\right]+\lambda \mathbb{E}\left[\left(u_{i n}-u_{i}\right)^{2}\right]\right) / 2,
$$

where $u_{i n} \equiv x_{i n}-\tilde{x}_{n}$ and $u_{i} \equiv x_{i}-\tilde{x}$ (this follows as in the proof of Proposition 3 in Vives (2011a)). We already know from the proof of part (i)(b) that $n \mathbb{E}\left[\left(\tilde{x}-\tilde{x}_{n}\right)^{2}\right] \stackrel{n}{\longrightarrow} \mathrm{AV} /(\beta+\lambda)^{2}$. We also know that $u_{i n}=\left(\tilde{t}_{n}-t_{i n}\right) /\left(\lambda+d_{n}\right)$ and $u_{i}=\left(\tilde{\theta}-t_{i}\right) / \lambda$, where $\tilde{t}_{n} \equiv \mathbb{E}\left[\tilde{\theta}_{n} \mid \tilde{s}_{n}\right]$ $t_{\text {in }} \equiv \mathbb{E}\left[\theta_{i} \mid s_{i}, \tilde{s}_{n}\right]=$ $\bar{\theta}+\frac{(1-\rho) \sigma_{\theta}^{2}}{\left(\sigma_{\theta}^{2}(1-\rho)+\sigma_{\varepsilon}^{2}\right)}\left(s_{i}-\bar{\theta}\right)+\frac{\sigma_{\varepsilon}^{2} \sigma_{\theta}^{2} \rho n}{\left(((n-1) \rho+1) \sigma_{\theta}^{2}+\sigma_{\varepsilon}^{2}\right)\left(\sigma_{\theta}^{2}(1-\rho)+\sigma_{\varepsilon}^{2}\right)}\left(\tilde{s}_{n}-\bar{\theta}\right)$, and $t_{i} \equiv \mathbb{E}\left[\theta_{i} \mid s_{i}, \tilde{\theta}\right]=\varsigma s_{i}+(1-\varsigma) \tilde{\theta}$ for $\varsigma=\left(1+\frac{\sigma_{\varepsilon}^{2}}{(1-\rho) \sigma_{\theta}^{2}}\right)^{-1}$. As a result, $\mathbb{E}\left[\left(u_{i n}-u_{i}\right)^{2}\right]=\frac{1}{\left(\lambda+d_{n}\right)^{2} \lambda^{2}}\left(\frac{(1-\rho) \sigma_{\theta}^{2}}{\left(\sigma_{\theta}^{2}(1-\rho)+\sigma_{\varepsilon}^{2}\right)}\right)^{2} \mathbb{E}\left[\left(\left(\tilde{s}_{n}-\tilde{\theta}\right) \lambda-\left(\tilde{\theta}-s_{i}\right) d_{n}\right)^{2}\right]$.

Further computations yield

$$
\mathbb{E}\left[\left(\left(\tilde{s}_{n}-\tilde{\theta}\right) \lambda-\left(\tilde{\theta}-s_{i}\right) d_{n}\right)^{2}\right]=n^{-1}\left(\sigma_{\theta}^{2}(1-\rho)+\sigma_{\varepsilon}^{2}\right)\left(2 \lambda d_{n}+\lambda^{2}+n d_{n}^{2}\right) .
$$

Therefore,

$$
\mathbb{E}\left[\left(u_{i n}-u_{i}\right)^{2}\right]=\frac{\left(2 \lambda d_{n}+\lambda^{2}+n d_{n}^{2}\right)(1-\rho)^{2} \sigma_{\theta}^{4}}{\left(\sigma_{\theta}^{2}(1-\rho)+\sigma_{\varepsilon}^{2}\right)\left(\lambda+d_{n}\right)^{2} \lambda^{2} n}
$$

since $d_{n}$ is of order $1 / n$, we have

$$
\lim _{n \rightarrow \infty} n \frac{\lambda \mathbb{E}\left[\left(u_{i n}-u_{i}\right)^{2}\right]}{2}=\frac{(1-\rho)^{2} \sigma_{\theta}^{4}}{2 \lambda\left(\sigma_{\theta}^{2}(1-\rho)+\sigma_{\varepsilon}^{2}\right)} .
$$

It follows that 


$$
n \mathrm{WL}_{n} \rightarrow \frac{\mathrm{AV}}{2(\beta+\lambda)}+\frac{(1-\rho)^{2} \sigma_{\theta}^{4}}{2 \lambda\left(\sigma_{\theta}^{2}(1-\rho)+\sigma_{\varepsilon}^{2}\right)}
$$

Illustration of equilibrium in the finite market and convergence properties with inelastic supply

Consider the case of an inelastic per capita supply of $q$ in Section 7.1. Suppose that traders $j \neq i$ employ linear strategies, $X\left(s_{j}, p\right)=b+a s_{j}-c p$. Then the marketclearing condition, $\sum_{j \neq i} X\left(s_{j}, p\right)+x_{i}=n q, c \neq 0$, implies that trader $i$ faces a residual inverse supply: $\quad p=I_{i}+d x_{i}, \quad$ where $\quad d=((n-1) c)^{-1} \quad$ and $I_{i}=d\left((n-1) b+a \sum_{j \neq i} s_{j}-q n\right)$. The (endogenous) parameter $d$ is the slope of inverse residual supply and the wedge introduced by market power. All the information that the price provides to trader $i$ about the signals of others is contained in the intercept $I_{i}$. The information available to trader $i$ is $\left\{s_{i}, p\right\}$ or, equivalently, $\left\{s_{i}, I_{i}\right\}$. Trader $i$ chooses $x_{i}$ to maximize

$$
\mathbb{E}\left[\pi_{i} \mid s_{i}, p\right]=x_{i}\left(\mathbb{E}\left[\theta_{i} \mid s_{i}, p\right]-p\right)-\frac{\lambda}{2} x_{i}^{2}=x_{i}\left(\mathbb{E}\left[\theta_{i} \mid s_{i}, p\right]-I_{i}-d x_{i}\right)-\frac{\lambda}{2} x_{i}^{2}
$$

The first-order condition (FOC) is $\mathbb{E}\left[\theta_{i} \mid s_{i}, p\right]-p=(d+\lambda) x_{i}$. An equilibrium requires that $d>0 .{ }^{41} \mathrm{~A}$ trader bids according to $p=\mathbb{E}\left[\theta_{i} \mid s_{i}, p\right]-(d+\lambda) x_{i}$, and competitive bidding obtains when $d=0$. A buyer $\left(x_{i}>0\right)$ underbids, $p<\mathbb{E}\left[\theta_{i} \mid s_{i}, p\right]-\lambda x_{i} ;$ since $d>0$, a seller $\left(x_{i}<0\right)$ overbids, $p>\mathbb{E}\left[\theta_{i} \mid s_{i}, p\right]-\lambda x_{i}$.

From the FOC and the Gaussian updating formulas for $\mathbb{E}\left[\theta_{i} \mid s_{i}, p\right]$, we immediately obtain the coefficients of the linear equilibrium strategy:

41 The second-order sufficient condition is fulfilled when $d>0$. 


$$
X_{n}\left(s_{i}, p\right)=b_{n}+a_{n} s_{i}-c_{n} p, \quad c_{n}=\frac{n-2-M_{n}}{\lambda(n-1)\left(1+M_{n}\right)},
$$

where

$$
M_{n} \equiv \frac{\rho \sigma_{\varepsilon}^{2} n}{(1-\rho)\left(\sigma_{\varepsilon}^{2}+(1+(n-1) \rho) \sigma_{\theta}^{2}\right)} \quad \text { and } \quad a_{n}=\frac{(1-\rho) \sigma_{\theta}^{2}}{\left(\sigma_{\varepsilon}^{2}+(1-\rho) \sigma_{\theta}^{2}\right)}\left(d_{n}+\lambda\right)^{-1}
$$

for $d_{n}=\left((n-1) c_{n}\right)^{-1}$. We require $n-2-M_{n}>0$ in order to guarantee the existence of an equilibrium (i.e., to obtain $d_{n}>0$ and $c_{n}>0$ ). (Observe that the inequality is always fulfilled for $n$ large because $M_{n}$ is bounded.) The reason for this requirement is that, if the inequality does not hold, then traders will seek to exploit their market power by submitting vertical schedules, and that is incompatible with the existence of equilibrium when there is no elastic exogenous supply.

The equilibrium price $p_{n}$ reveals the average signal $\tilde{s}_{n} ;$ therefore, $\mathbb{E}\left[\theta_{i} \mid s_{i}, p_{n}\right]=\mathbb{E}\left[\theta_{i} \mid s_{i}, \tilde{s}_{n}\right]$ and $n^{-1} \sum_{i=1}^{n} \mathbb{E}\left[\theta_{i} \mid s_{i}, \tilde{s}_{n}\right]=\mathbb{E}\left[\tilde{\theta}_{n} \mid \tilde{s}_{n}\right]$. Averaging the FOCs, we obtain that $\mathbb{E}\left[\tilde{\theta}_{n} \mid \tilde{s}_{n}\right]-p_{n}=\left(d_{n}+\lambda\right) \tilde{x}_{n}=\left(d_{n}+\lambda\right) q \quad$ and $\quad$ hence $p_{n}=\mathbb{E}\left[\tilde{\theta}_{n} \mid \tilde{s}_{n}\right]-\left(d_{n}+\lambda\right) q$.

We have that $X_{n}\left(s_{i}, p\right) \stackrel{n}{\longrightarrow}(1-\lambda a) q+a\left(s_{i}-p\right)$, the trading strategy in the inelastic supply case in the limit economy. ${ }^{42}$ Furthermore, $p_{n} \stackrel{n}{\longrightarrow} p=\tilde{\theta}-\lambda q$ in mean square at the rate $1 / \sqrt{n}$. This follows because $d_{n} \stackrel{n}{\longrightarrow} 0$ and $\mathbb{E}\left[\tilde{\theta}_{n} \mid \tilde{s}_{n}\right] \stackrel{n}{\longrightarrow} \tilde{\theta}$ in mean square (given that $\tilde{\theta}_{n} \stackrel{n}{\longrightarrow} \tilde{\theta}$ and $\left(\sum_{i} \varepsilon_{i}\right) / n \stackrel{n}{\longrightarrow} 0$ in mean square, both at rate $1 / \sqrt{n}$ ). In fact, we have $n \mathbb{E}\left[\left(\tilde{\theta}-\mathbb{E}\left[\tilde{\theta}_{n} \mid \tilde{s}_{n}\right]\right)^{2}\right] \stackrel{n}{\longrightarrow} \mathrm{AV}$, where $\mathrm{AV}=(1-\rho) \sigma_{\theta}^{2}+\sigma_{\varepsilon}^{2}$ if $\rho>0$

42 This statement is proved as follows: $c_{n} \stackrel{n}{\longrightarrow} a \equiv \lambda^{-1}(M+1)^{-1}$ if $\rho>0$ (since then $M_{n} \stackrel{n}{\longrightarrow} M$ ), and $\quad c_{n} \stackrel{n}{\longrightarrow} \lambda^{-1}$ if $\rho=0$ (since then $M_{n}=0$ ); furthermore, $a_{n} \stackrel{n}{\longrightarrow} a$ because $d_{n}=\left((n-1) c_{n}\right)^{-1} \stackrel{n}{\longrightarrow} 0$. It can be checked similarly that $b_{n} \stackrel{n}{\longrightarrow}(1-\lambda a) q$. 
and $\mathrm{AV}=\sigma_{\theta}^{4}\left(\sigma_{\theta}^{2}+\sigma_{\varepsilon}^{2}\right)^{-1}$ if $\rho=0$. This means that the convergence is faster (in terms of asymptotic variance) the closer we are to the common value case, the less prior uncertainty there is, and the less noisy are the signals (if $\rho>0$ ). ${ }^{43}$ The market power distortion $d_{n}=\left((n-1) c_{n}\right)^{-1}$ (i.e., the amount of over- or underbidding) is of the order $1 / n$.

Proof of Proposition 3: We want to analyze symmetric equilibria. Note that for $\left(\tau_{\varepsilon_{i}}, X_{i}(\cdot, \cdot)\right)_{i=1, \ldots n}$ to be an equilibrium, $\left(X_{i}(\cdot, \cdot)\right)_{i=1, \ldots n}$ has to be a DFE for given precisions $\left(\tau_{\varepsilon_{i}}\right)_{i=1, \ldots n}$. We prove (ii) first. Suppose traders $j \neq i$ have information precision $\tau_{\varepsilon}>0$ and use identical strategies based on linear demand schedules with coefficients $(b, a, c)$. Trader $i$ has precision $\tau_{\varepsilon_{i}}$ and his optimal demand schedule is $X_{i}\left(s_{i}, p\right)=\left(\mathbb{E}\left[\theta_{i} \mid s_{i}, p\right]-p\right)\left(d_{i}+\lambda\right)^{-1}$ where $d_{i}=\left(\beta^{-1} n+(n-1) c\right)^{-1}$. The expected profits of trader $i$ are a function of $\left(b, a, c, \tau_{\varepsilon}, \tau_{\varepsilon_{i}}\right)$ and can be checked to be strictly concave in $\tau_{\varepsilon_{i}}$ for $d>0$. If we let $d_{n}=\left(\beta^{-1} n+(n-1) c_{n}\right)^{-1}$, where $c_{n}$ is the symmetric solution when $\tau_{\varepsilon_{i}}=\tau_{\varepsilon}$ and $\rho<1$ (given by Proposition 1 in Vives (2011a), then it follows from Section S.4 in Vives (2011b) that

$$
\left.\psi_{n}\left(\tau_{\varepsilon}\right) \equiv \frac{\partial \mathbb{E}\left[\pi_{i}\right]}{\partial \tau_{\varepsilon_{i}}}\right|_{\tau_{\varepsilon_{i}}=\tau_{\varepsilon}}=\frac{1}{2\left(2 d_{n}+\lambda\right)} \frac{\left(\tau_{\varepsilon}(1-\rho)(1+\rho(n-1))+\tau_{\theta}\right)^{2}}{\left(\tau_{\varepsilon}(1-\rho)+\tau_{\theta}\right)^{2}\left(\tau_{\varepsilon}(1+\rho(n-1))+\tau_{\theta}\right)^{2}} .
$$

Interior symmetric equilibria for information precision are characterized by the solution of $\psi_{n}\left(\tau_{\varepsilon}\right)-H^{\prime}\left(\tau_{\varepsilon}\right)=0$. An interior symmetric equilibrium with $\tau_{\varepsilon}^{*}(n)>0$ exists if $H^{\prime}(0)<\psi_{n}(0) \quad$ where $\quad \psi_{n}(0) \equiv \lim _{\tau_{\varepsilon} \rightarrow 0} \psi_{n}\left(\tau_{\varepsilon}\right)=\left(2\left(2 d_{n}(0)+\lambda\right) \tau_{\theta}^{2}\right)^{-1} \quad$ with $d_{n}(0) \equiv \lim _{\tau_{\varepsilon} \rightarrow 0} d_{n}\left(\tau_{\varepsilon}\right)=\left(n \beta^{-1}+(n-1) \widehat{c}_{n}\right)^{-1}$ where $\widehat{c}_{n}=\lim _{\tau_{\varepsilon} \rightarrow 0} c_{n}$ (see Section S.4 in Vives

43 If $\rho=0$ then $\tilde{\theta}=\bar{\theta}$; in this case, more noise in the signals makes $\mathbb{E}\left[\tilde{\theta}_{n} \mid \tilde{s}_{n}\right]$ closer to $\bar{\theta}$, which speeds up convergence. 
(2011b)). For $n$ large, $\psi_{n}\left(\tau_{\varepsilon}\right)$ is strictly decreasing in $\tau_{\varepsilon}$. It follows that for $n$ large there is a unique symmetric equilibrium $\tau_{\varepsilon}^{*}(n)>0$, the unique solution to $\phi_{n}\left(\tau_{\varepsilon}\right) \equiv \psi_{n}\left(\tau_{\varepsilon}\right)-H^{\prime}\left(\tau_{\varepsilon}\right)=0$, if and only if $H^{\prime}(0)<\psi_{n}(0)$.

We prove (i) here. Similarly as before, suppose traders $j \neq i$ have information precision $\tau_{\varepsilon}=0$ and use identical strategies based on linear demand schedules with coefficients $(b, c)$. Trader $i$ has precision $\tau_{\varepsilon_{i}}$ and his optimal demand schedule is $X_{i}\left(s_{i}, p\right)=\left(\mathbb{E}\left[\theta_{i} \mid s_{i}, p\right]-p\right)\left(d_{i}+\lambda\right)^{-1}$ where $d_{i}=\left(\beta^{-1} n+(n-1) c\right)^{-1}$. The expected profits of trader $i$ are a function of $\left(b, c, \tau_{\varepsilon_{i}}\right), \mathbb{E}\left[\pi_{i}\right]=\left((\lambda / 2)+d_{i}\right)\left(\left(\mathbb{E}\left[x_{i}\right]\right)^{2}+\operatorname{var}\left[x_{i}\right]\right)$ where

$$
\operatorname{var}\left[x_{i}\right]=\frac{\tau_{\varepsilon_{i}}}{\left(\lambda+2 d_{i}\right)^{2}\left(\tau_{\theta}+\tau_{\varepsilon_{i}}\right) \tau_{\theta}} .
$$

Since $\mathbb{E}\left[x_{i}\right]$ depends only on $\left(b, c, d_{i}\right)$ and $d_{i}$ only on $c$ it follows that

$$
\left.\frac{\partial \mathbb{E}\left[\pi_{i}\right]}{\partial \tau_{\varepsilon_{i}}}\right|_{\substack{\tau_{\varepsilon_{j}}=0 \\ j \neq i}}=\frac{1}{2\left(2 d_{i}+\lambda\right)} \frac{1}{\left(\tau_{\varepsilon_{i}}+\tau_{\theta}\right)^{2}}
$$

and $\mathbb{E}\left[\pi_{i}\right]$ is strictly concave in $\tau_{\varepsilon_{i}}$. If we evaluate $d_{i}$ at the candidate symmetric no information equilibrium $d_{i}=d_{n}^{f}$ we obtain

$$
\left.\varphi_{n}\left(\tau_{\varepsilon_{i}}\right) \equiv \frac{\partial \mathbb{E}\left[\pi_{i}\right]}{\partial \tau_{\varepsilon_{i}}}\right|_{\substack{c_{\varepsilon_{j}}=0 \\ j \neq i}}=\frac{1}{2\left(2 d_{n}^{f}+\lambda\right)} \frac{1}{\left(\tau_{\varepsilon_{i}}+\tau_{\theta}\right)^{2}} .
$$

We have that $\varphi_{n}\left(\tau_{\varepsilon_{i}}\right)$ is strictly decreasing in $\tau_{\varepsilon_{i}}$. Therefore, a symmetric equilibrium with $\tau_{\varepsilon}^{*}(n)=0$ exists if and only if $\varphi_{n}(0) \leq H^{\prime}(0)$, where $\varphi_{n}(0)=\left(2\left(2 d_{n}^{f}+\lambda\right) \tau_{\theta}^{2}\right)^{-1}$. 
(iii) We have that $d_{n}(0)>d_{n}^{f}$ for $\rho>0$ and $d_{n}(0)=d_{n}^{f}$ for $\rho=0$. This follows comparing the expressions (see Vives (2011a)) for $d_{n}^{f}=\frac{\beta}{n}-\frac{1}{2}(\beta+\lambda)+\frac{1}{2 n} \sqrt{\beta^{2}(n-2)^{2}+2 n^{2} \beta \lambda+n^{2} \lambda^{2}}$ and $d_{n}(0)=\frac{\beta\left(\frac{\rho}{1-\rho}-1+\frac{2}{n}\right)-\lambda\left(\frac{\rho}{1-\rho}+1\right)+\sqrt{\beta^{2}\left(\frac{\rho}{1-\rho}-1+\frac{2}{n}\right)^{2}+2 \beta \lambda\left(\frac{\rho}{1-\rho}+1\right)^{2}+\lambda^{2}\left(\frac{\rho}{1-\rho}+1\right)^{2}}}{\frac{2}{1-\rho}}$.

Therefore, $\varphi_{n}(0)=\left(2\left(2 d_{n}^{f}+\lambda\right) \tau_{\theta}^{2}\right)^{-1}>\psi_{n}(0)=\left(2\left(2 d_{n}(0)+\lambda\right) \tau_{\theta}^{2}\right)^{-1}$ for $\rho>0$. In the region $\psi_{n}(0) \leq H^{\prime}(0)<\varphi_{n}(0)$ there is no symmetric equilibrium for $n$ large since neither an equilibrium with information acquisition nor without obtains.

Proof of Proposition 4: (i) Observe that, since $d_{n} \stackrel{n}{\longrightarrow} 0$ for $\tau_{\varepsilon}>0$, we have then

$$
\psi_{n}\left(\tau_{\varepsilon}\right) \stackrel{n}{\longrightarrow} \psi_{\infty}\left(\tau_{\varepsilon}\right) \equiv \frac{(1-\rho)^{2}}{2 \lambda\left(\tau_{\theta}+(1-\rho) \tau_{\varepsilon}\right)^{2}} .
$$

We know that if $\rho \in\left[0,1-\tau_{\theta} \sqrt{2 \lambda H^{\prime}(0)}\right)$, there is a unique $\tau_{\varepsilon}^{*}>0$ which solves

$$
\phi\left(\tau_{\varepsilon}\right)=\frac{(1-\rho)^{2}}{2 \lambda\left(\tau_{\theta}+(1-\rho) \tau_{\varepsilon}\right)^{2}}-H^{\prime}\left(\tau_{\varepsilon}\right)=0 .
$$

We conclude that $\tau_{\varepsilon}^{*}(n) \rightarrow \tau_{\varepsilon}^{*}>0\left(\right.$ where $\left.\phi\left(\tau_{\varepsilon}^{*}\right)=0\right)$ as $n \rightarrow \infty$. The convergence result follows since when at the $n$-replica we have $\tau_{\varepsilon}^{*}(n)>0$, then

$$
\mathbb{E}\left[\left(\tilde{\theta}-\mathbb{E}\left[\tilde{\theta}_{n} \mid \tilde{s}_{n}\right]\right)^{2}\right]=\frac{\sigma_{\theta}^{2}(1-\rho)(\rho(n-1)+1)+n \rho\left(\tau_{\varepsilon}^{*}(n)\right)^{-1}}{n\left(\sigma_{\theta}^{2}+\left(\tau_{\varepsilon}^{*}(n)\right)^{-1}+(n-1) \rho \sigma_{\theta}^{2}\right)} \sigma_{\theta}^{2}
$$

and therefore $n \mathbb{E}\left[\left(\tilde{\theta}-\mathbb{E}\left[\tilde{\theta}_{n} \mid \tilde{s}_{n}\right]\right)^{2}\right] \stackrel{n}{\longrightarrow} \mathrm{AV}^{*}$, where $\mathrm{AV}^{*}=(1-\rho) \sigma_{\theta}^{2}+\left(\tau_{\varepsilon}^{*}\right)^{-1}$ if $\rho>0$ and $\mathrm{AV}^{*}=\sigma_{\theta}^{4}\left(\sigma_{\theta}^{2}+\left(\tau_{\varepsilon}^{*}\right)^{-1}\right)^{-1}$ if $\rho=0$ since $\tau_{\varepsilon}^{*}(n) \rightarrow \tau_{\varepsilon}^{*}>0$. 
(ii) We have that $\varphi_{n}(0) \rightarrow\left(2 \lambda \tau_{\theta}^{2}\right)^{-1}$ from below $\left(\varphi_{n}(0) \leq\left(2 \lambda \tau_{\theta}^{2}\right)^{-1}\right)$ since $d_{n}^{f}>0$ and $d_{n}^{f} \rightarrow 0$ as $n \rightarrow \infty$. It follows that if $\bar{\rho} \leq 0, \tau_{\varepsilon}^{*}(n)=0$ for any $n$ since for $\bar{\rho} \leq 0$ we have that $\varphi_{n}(0) \leq\left(2 \lambda \tau_{\theta}^{2}\right)^{-1} \leq H^{\prime}(0)$.

Claim 1. (i) $\lim _{n \rightarrow \infty} \lim _{\tau_{\varepsilon} \rightarrow 0} d_{n}\left(\tau_{\varepsilon}\right)=\lim _{n \rightarrow \infty} d_{n}(0+)>0 \quad$ except $\quad$ if $\quad \beta \rho \lambda=0 \quad$ and $\lim _{\rho \rightarrow 1} \lim _{n \rightarrow \infty} d_{n}(0+)=\lim _{n \rightarrow \infty} \lim _{\rho \rightarrow 1} d_{n}(0+)=\beta$. (ii) There is a unique value of $\beta, \hat{\beta}>0$, such that $\lim _{n \rightarrow \infty} \psi_{n}(0)>(1-\rho)^{2}\left(2 \lambda \tau_{\theta}^{2}\right)^{-1}$ if and only if $\beta<\hat{\beta}$, and $\hat{\beta} \rightarrow \infty$ as $\rho \rightarrow 1$.

Proof: $\quad$ (i) $\quad \mathrm{We} \quad$ obtain that $\lim _{n \rightarrow \infty} d_{n}(0)=\left(\beta(2 \rho-1)-\lambda+\sqrt{\beta^{2}(2 \rho-1)^{2}+2 \beta \lambda+\lambda^{2}}\right) / 2$, which is positive for $\beta \rho \lambda>0$ and 0 if $\beta \rho=0$. (ii) The result follows analyzing the expressions for $\lim _{n \rightarrow \infty} \psi_{n}(0)=\left(2\left(2 \lim _{n \rightarrow \infty} d_{n}(0)+\lambda\right) \tau_{\theta}^{2}\right)^{-1}$ and $\lim _{n \rightarrow \infty} d_{n}(0)$.

Proof of Proposition 5: From Claim 1 (ii) we have that if $\beta<\hat{\beta}$ then $\lim _{n \rightarrow \infty} \psi_{n}(0)>(1-\rho)^{2}\left(2 \lambda \tau_{\theta}^{2}\right)^{-1} \quad$. Proposition 3 implies that if $\left(2\left(2 \lim _{n \rightarrow \infty} d_{n}(0)+\lambda\right) \tau_{\theta}^{2}\right)^{-1}>H^{\prime}(0)$ then $\tau_{\varepsilon}^{*}(n)>0$ for $n$ large and there is no equilibrium in the continum economy since $\left(2 \lambda \tau_{\theta}^{2}\right)^{-1} \geq\left(2\left(2 \lim _{n \rightarrow \infty} d_{n}(0)+\lambda\right) \tau_{\theta}^{2}\right)^{-1}>H^{\prime}(0) \geq(1-\rho)^{2}\left(2 \lambda \tau_{\theta}^{2}\right)^{-1}$ (in particular, $\tau_{\varepsilon}^{*}=0$ is not an equilibrium). Suppose that $\lim _{n \rightarrow \infty} \tau_{\varepsilon}^{*}(n)=\tau_{\varepsilon}>0$. Since for $\tau_{\varepsilon}>0$ we have that $\psi_{n}\left(\tau_{\varepsilon}\right) \stackrel{n}{\longrightarrow} \psi_{\infty}\left(\tau_{\varepsilon}\right)$, we should have $\tau_{\varepsilon}=\tau_{\varepsilon}^{*}$ but we know there is no equilibrium in the continuum economy. Therefore, $\lim _{n \rightarrow \infty} \tau_{\varepsilon}^{*}(n)=0$. Furthermore, for any $n$, as $\rho \rightarrow 1$ we have that $\widehat{c}_{n} \rightarrow-1 / \beta, d_{n}(0) \rightarrow \beta$, and $\psi_{n}(0) \rightarrow\left(2(2 \beta+\lambda) \tau_{\theta}^{2}\right)^{-1}$. The result for $\rho$ close to 1 follows. 


\section{References}

Admati, Anat (1985). “A Noisy Rational Expectations Equilibrium for Multi-Asset Securities Markets." Econometrica, 53, 629-657.

Allen, Beth (1981). "A Class of Monotone Economies in which Rational Expectations Equilibria Exist but Prices Do Not Reveal All Information.” Economics Letters, 7, 227-232.

Allen, Jason, Ali Hortaçsu, and Jakub Kastl (2011). "Crisis Management: Analyzing Default Risk and Liquidity Demand during Financial Stress.” mimeo.

Ausubel, Lawrence (1990). "Partially-revealing Rational-expectations Equilibrium in a Competitive Economy." Journal of Economic Theory, 50, 93-126.

Amador, Manuel and Pierre-Olivier Weill (2010). "Public Communication and Welfare"; Journal of Political Economy, 118, 866-907.

Anderson, Robert and Hugo Sonnenschein (1982). "On the Existence of Rational Expectations Equilibrium.” Journal of Economic Theory, 26, 261-278.

Beja, Avraham (1977). "The Limits of Price Information in Market Processes.” Institute of Business and Economic Research, University of California Working paper no. 61.

Bergemann, Dirk, Tibor Heumann, and Stephen Morris (2014). "Information and Market Power in Rational Expectations Equilibrium.” mimeo.

Black, Fischer (1986). "Noise.” The Journal of Finance, 41, 529-543.

Blume, Lawrence and David Easley (1990). "Implementation of Walrasian Expectations Equilibria." Journal of Economic Theory, 51, 207-227.

Cassola, Nuno, Ali Hortaçsu, and Jakub Kastl (2013). "The 2007 Subprime Market Crisis Through the Lens of the European Central Bank Auctions for Short-term Funds." Econometrica, 81, 1309-1345.

Condie, Scott and Jayant Ganguli (2011). "Ambiguity and Rational Expectations Equilibria." Review of Economic Studies, 78, 821-845. 
Cripps, Martin and Jeroen Swinkels (2006). "Efficiency of Large Double Auctions." Econometrica, 74, 47-92.

Diamond, Douglas and Robert Verrecchia (1981). "Information Aggregation in a Noisy Rational Expectations Economy.” Journal of Financial Economics, 9, 221-235.

Dubey, Pradeep, John Geneakoplos, and Martin Shubik (1987). "The Revelation of Information in Strategic Market Games: A Critique of Rational Expectations Equilibrium.” Journal of Mathematical Economics, 16, 105-138.

Ewerhart, Christian, Nuno Cassola, and N. Valla (2010). "Declining Valuations and Equilibrium Bidding in Central Bank Refinancing Operations." International Journal of Industrial Organization, 28, 30-43.

Ganguli, Jayant and Liyan Yang (2009). "Complementarities, Multiplicity, and Supply Information.” Journal of the European Economic Association, 7, 90-115.

Golosov, Mikhail, Guido Lorenzoni, and Aleh Tsyvinski (2014). "Decentralized Trading with Private Information.” Econometrica, 82, 1055-1091.

Grossman, Sandford (1981). "An Introduction to the Theory of Rational Expectations under Asymmetric Information." Review of Economic Studies, 48, 541-559.

Grossman, Sandford and Joseph Stiglitz (1976). "Information and Competitive Price Systems.”.American Economic Review, 66, 246-253.

Grossman, Sandford and Joseph Stiglitz (1980). “On the Impossibility of Informationally Efficient Markets." American Economic Review, 70, 393-408.

Gul, Faruk and Andrew Postlewaite (1992). “Asymptotic Efficiency in Large Exchange Economies with Assymetric Information.” Econometrica, 60, 1273-1292.

Hayek, Friedrich (1945). "The Use of Knowledge in Society." American Economic Review, 35, 519-530.

Heider, Florian, Marie Hoerova, and Cornelia Holthausen (2009). "Liquidity Hoarding and Interbank Market Spreads: The Role of Counterparty Risk.” mimeo.

Hellwig, Martin (1980). "On the Aggregation of Information in Competitive Markets." Journal of Economic Theory, 22, 477-498. 
Hellwig, Martin (1982). "Rational Expectations Equilibrium with Conditioning on Past Prices: A Mean-Variance Example.” Journal of Economic Theory, 26, 279-312.

Hortaçsu, Ali and Jakub Kastl (2012). "Valuing Dealers' Informational Advantage: A Study of Canadian Treasury Auctions.” Econometrica, 80, 2511-2542.

Jackson, Matthew (1991). "Equilibrium, Price Formation, and the Value of Private Information." Review of Financial Studies, 4, 1-16.

Jackson, Matthew (2003). "Efficiency and Information Aggregation in Auctions with Costly Information.” Review of Economic Design, 8, 121-141.

Krebs, Thomas (2007). "Rational Expectations Equilibrium and the Strategic Choice of Costly Information.” Journal of Mathematical Economics, 43, 532-548.

Kovalenkov, Alexander and Xavier Vives (2014). "Competitive Rational Expectations Equilibria without Apology." Journal of Economic Theory, 149, 211-235.

Kyle, Albert (1989). "Informed Speculation with Imperfect Competition." Review of Economic Studies, 56, 317-356.

Linzert, Tobias, Dieter Nautz, and Ulrich Bindseil (2007). "Bidding Behavior in the Longer Term Refinancing Operations of the European Central Bank: Evidence from a Panel Sample Selection Model.” Journal of Banking \& Finance, 31, 1521-1543.

Manzano, Carolina and Xavier Vives (2011). "Public and Private Learning from Prices, Strategic Substitutability and Complementarity, and Equilibrium Multiplicity." Journal of Mathematical Economics, 47, 346-369.

Mas-Colell, Andreu and Xavier Vives (1993). "Implementation in Economies with a Continuum of Agents." Review of Economic Studies, 60, 613-629.

Matthews, Steven (1984). "Information Acquisition in Discriminatory Auctions," in Bayesian Models in Economic Theory, (M. Boyer and R. Kihlstrom, eds.), Elsevier Science Pub., 181-207.

Mele, Antonio and Francesco Sangiorgi (2009). “Ambiguity, Information Acquisition and Price Swings in Asset Markets." FMG discussion paper series no. 633. 
Milgrom, Paul (1981). "Rational Expectations, Information Acquisition, and Competitive Bidding." Econometrica, 49, 921-943.

Muendler, Marc-Andreas (2007). "The Possibility of Informationally Efficient Markets." Journal of Economic Theory, 133, 467-483.

Palfrey, Thomas (1985). "Uncertainty Resolution, Private Information Aggregation and the Cournot Competitive Limit." Review of Economic Studies, 52, 168, 69-74.

Palfrey, Thomas and Sanjay Srivastava (1986). "Private Information in Large Economies." Journal of Economic Theory, 39, 34-58.

Pesendorfer, Wolfgang and Jeroen Swinkels (1997). "The Loser's Curse and Information Aggregation in Common Value Auctions." Econometrica, 65, 1247-1281.

Postlewaite, Andrew and David Schmeidler (1986). "Implementation in Differential Information Economies.” Journal of Economic Theory, 39, 14-33.

Reny, Philip and Martin Perry (2006). "Toward a Strategic Foundation for Rational Expectations Equilibrium.” Econometrica, 74, 1231-1269.

Rostek, Marzena and Marek Weretka (2012). "Price Inference in Small Markets." Econometrica, 80, 687-711.

Rustichini, Aldo, Mark Satterthwaite and Stephen Williams (1994). "Convergence to Efficiency in a Simple Market with Incomplete Information.” Econometrica, 62, 1041-1063.

Satterthwaite, Mark and Stephen Williams (1989). "The Rate of Convergence to Efficiency in the Buyer's Bid Double Auction as the Market Becomes Large." Review of Economic Studies, 56, 477-498.

Verrecchia, Robert (1982). "Information Acquisition in a Noisy Rational Expectations Economy." Econometrica, 50, 1415-1430.

Vives, Xavier (1988). "Aggregation of Information in Large Cournot Markets." Econometrica, 56, 4, 851-876.

Vives, Xavier (2010). "Asset Auctions, Information, and Liquidity." Journal of the European Economic Association, 8, 467-477. 
Vives, Xavier (2011a). "Strategic Supply Function Competition with Private Information." Econometrica, 79, 1919-1966.

Vives, Xavier (2011b). "Supplement to 'Strategic Supply Function Competition With

Private Information: Proofs, Simulations, and Extensions.” Econometrica Supplemental Material, 79, http://www.econometricsociety.org/ecta/Supmat/8126_proofs.pdf.

Vives, Xavier (2013). "Endogenous Public Information and Welfare in Market Games." CEPR Discussion Paper 8437.

Wang, J. and J. Zender (2002). “Auctioning Divisible Goods.” Economic Theory, 19, 673-705.

Wilson, Robert (1977). “A Bidding Model of Perfect Competition.” Review of Economic Studies, 44, 511-518.

Wilson, Robert (1979). "Auction of Shares.” Quarterly Journal of Economics, 93, 675689.

Wilson, Robert (1985). "Incentive Efficiency of Double Auctions." Econometrica, 53, 1233-1243. 